\title{
Coupled reaction-diffusion processes inducing an evolution of the microstructure: analysis and homogenization
}

\author{
Malte A. Peter* \\ Centre for Industrial Mathematics, FB 3, University of Bremen, Germany
}

\begin{abstract}
Chemical processes in porous media often cause a change of the microstructure of the porous material due to interaction with the solid matrix, by reaction or adsorption, e.g. We consider a reaction-diffusion problem where a solid matrix constituent is converted into another one of different density. Thus, the solid matrix locally grows or shrinks in volume, which in turn changes the pore-air volume. This affects the transport of reactants in the pore air. The homogenization of this problem with evolving microstructure is performed using the method of transformation to a periodic reference domain, which has recently been put forward by the author. The final system to be homogenized consists of three coupled partial differential equations for the concentrations coupled to one ordinary differential equation for a quantity describing the evolution of the pore-air volume.
\end{abstract}

Key words: Homogenization, evolution of the microstructure, reaction-diffusion system, concrete carbonation

\section{Introduction}

We consider the problem of reaction, diffusion and interfacial exchange of two species in a porous medium $\Omega$ made up of three phases: pore air $\Omega^{\mathrm{a}}$, pore water $\Omega^{\mathrm{w}}$ and solid matrix $\Omega^{\mathrm{s}}$. The first species, A, diffuses through the pore air and dissolves in the pore water. The second species, $\mathrm{B}$, is part of the solid matrix

* Current address: Department of Mathematics, University of Auckland, Private Bag 92019, Auckland 1142, New Zealand. Phone: +6493737599x83974. Fax: +6493737457 .

Email address: mpeter@math.auckland.ac.nz (Malte A. Peter). 
and dissolves in the pore water. The two species may react in a reaction of the form

$$
\mathrm{A}+\mathrm{B} \longrightarrow \mathrm{C}+\ldots,
$$

where the reaction product $\mathrm{C}$ is of low solubility and can hence be associated with the solid matrix again. The special feature of the problem under consideration is that solid B is of highly different density compared to solid C so that the reaction causes a local growing or shrinking of the solid matrix. In turn, this changes the shape of pore air and pore water. Therefore, the volume occupied by the porous medium $\Omega$ is time-independent while its parts, $\Omega^{\mathrm{a}}, \Omega^{\mathrm{w}}$ and $\Omega^{\mathrm{s}}$, may evolve owing to the conversion of $\mathrm{B}$ to $\mathrm{C}$.

A particular application exhibiting these features is concrete carbonation. The main process of this chemical degradation mechanism can be described by atmospheric carbon dioxide (A) diffusing in the pore air of the concrete and dissolving in the pore water. There it reacts with certain concrete constituents, most importantly with calcium hydroxide $(\mathrm{B})$. Calcium carbonate $(\mathrm{C})$ is produced in this reaction, which causes a decrease of $\mathrm{pH}$ in the concrete and an increase of volume of solid matrix since the calcium carbonate takes up more volume than the reactant calcium hydroxide. The drop in $\mathrm{pH}$ facilitates the corrosion of reinforcing steel bars and thus decreases the service-life of the structure. More information about concrete carbonation can be found in [1,2] and in $[3,4]$ in the context of multi-scale approaches.

Denoting the mass concentration of $\mathrm{A}$ by $u$ and of $\mathrm{B}$ by $v$, the above problem can be described by

$$
\begin{array}{cc}
\partial_{t} u^{\mathrm{a}}(x, t)-\nabla \cdot\left(D^{\mathrm{a}} \nabla u^{\mathrm{a}}\right)=0, & x \in \Omega^{\mathrm{a}}(t), t \in S, \\
\partial_{t} u^{\mathrm{w}}(x, t)-\nabla \cdot\left(D^{\mathrm{w}} \nabla u^{\mathrm{w}}\right)=-r^{\mathrm{u}} f^{\mathrm{r}}, \quad x \in \Omega^{\mathrm{w}}(t), t \in S, \\
\partial_{t} v^{\mathrm{w}}(x, t)-\nabla \cdot\left(E^{\mathrm{w}} \nabla v^{\mathrm{w}}\right)=+r^{\mathrm{v}} f^{\mathrm{r}}, \quad x \in \Omega^{\mathrm{w}}(t), t \in S, \\
-\left(D^{\mathrm{a}} \nabla u^{\mathrm{a}}\right) \cdot n^{\mathrm{a}}=\left(D^{\mathrm{w}} \nabla u^{\mathrm{w}}\right) \cdot n^{\mathrm{w}}, & x \in \partial \Omega^{\mathrm{a}}(t) \cap \partial \Omega^{\mathrm{w}}(t), t \in S, \\
-\left(D^{\mathrm{a}} \nabla u^{\mathrm{a}}\right) \cdot n^{\mathrm{a}}=a\left(u^{\mathrm{a}}-u^{\mathrm{w}}\right), & x \in \partial \Omega^{\mathrm{a}}(t) \cap \partial \Omega^{\mathrm{w}}(t), t \in S, \\
-\left(D^{\mathrm{a}} \nabla u^{\mathrm{a}}\right) \cdot n^{\mathrm{a}}=0, & x \in \partial \Omega^{\mathrm{a}}(t) \cap \partial \Omega^{\mathrm{s}}(t), t \in S, \\
-\left(D^{\mathrm{w}} \nabla u^{\mathrm{w}}\right) \cdot n^{\mathrm{w}}=0, & x \in \partial \Omega^{\mathrm{w}}(t) \cap \partial \Omega^{\mathrm{s}}(t), t \in S, \\
-\left(E^{\mathrm{w}} \nabla v^{\mathrm{w}}\right) \cdot n^{\mathrm{w}}=0, & x \in \partial \Omega^{\mathrm{w}}(t), t \in S,
\end{array}
$$

where we have used superscripts a and $\mathrm{w}$ in order to distinguish quantities associated with pore air and pore water, respectively. We prescribe homogeneous Neumann boundary conditions at the exterior boundary $\partial \Omega$ for simplicity as well as initial conditions $\left(u^{\mathrm{a}}(0), u^{\mathrm{w}}(0), v^{\mathrm{w}}(0)\right)=\left(u_{0}^{\mathrm{a}}, u_{0}^{\mathrm{w}}, v_{0}^{\mathrm{w}}\right)$.

In order to upscale the problem using periodic homogenization, we want to employ the ideas of homogenization in domains with evolving microstructure described in [5], i.e. it is assumed that there exists a reference configuration, which is $\varepsilon$-periodic. By this, we mean that there exists a reference cell $Y=$ 
$(0,1)^{N}$ with $\bar{Y}=\bar{Z}^{\mathrm{a}} \cup \bar{Z}^{\mathrm{w}} \cup \bar{Z}^{\mathrm{s}}$ and $Z^{\mathrm{a}} \cap Z^{\mathrm{w}} \cap Z^{\mathrm{s}}=\emptyset$, such that $\Omega_{*}^{i}=$ $\bar{\Omega} \cap \operatorname{int} \bigcup_{k \in \mathbb{Z}^{N}} \varepsilon\left(\bar{Z}^{i}+k\right)$ is a reference configuration of $\Omega^{i}, i \in\{\mathrm{a}, \mathrm{w}, \mathrm{s}\}$.

For simplicity, we assume the geometry at $t=0$ coincides with this reference configuration. Furthermore, the evolution of the three parts of $\Omega$ can be described by orientation-preserving mappings $\psi_{\varepsilon}^{i}(\cdot, t): \Omega_{\varepsilon}^{i} \rightarrow \Omega^{i}(t), i \in\{\mathrm{a}, \mathrm{w}, \mathrm{s}\}$, for each $t \in S$. Here, we have written $\Omega_{\varepsilon}^{i}$ for $\Omega^{i}(0)=\Omega_{*}^{i}$ in order to emphasize the $\varepsilon$-periodicity of the geometry. For future use, we introduce the characteristic function of $Z^{i}, \chi^{i}, i \in\{\mathrm{a}, \mathrm{w}, \mathrm{s}\}$, and $\chi_{\varepsilon}^{i}(x)=\chi^{i}(x / \varepsilon)$ (having periodically extended $\chi^{i}$ to $\mathbb{R}^{N}$ ).

Defining $\Psi_{\varepsilon}^{i}=\nabla \psi_{\varepsilon}^{i}$ and $J_{\varepsilon}^{i}=\operatorname{det} \Psi_{\varepsilon}^{i}, i \in\{\mathrm{a}, \mathrm{w}, \mathrm{s}\}$, the system of equations (2) may be written in the reference configuration (cf. [5]):

$$
\begin{array}{cl}
\partial_{t}\left(J_{\varepsilon}^{\mathrm{a}} \hat{u}_{\varepsilon}^{\mathrm{a}}\right)-\nabla \cdot\left(J_{\varepsilon}^{\mathrm{a}} \Psi_{\varepsilon}^{\mathrm{a}-1} \hat{D}_{\varepsilon}^{\mathrm{a}} \Psi_{\varepsilon}^{\mathrm{a}-T} \nabla \hat{u}_{\varepsilon}^{\mathrm{a}}\right)=0, & x \in \Omega_{\varepsilon}^{\mathrm{a}}, t \in S, \\
\partial_{t}\left(J_{\varepsilon}^{\mathrm{w}} \hat{u}_{\varepsilon}^{\mathrm{w}}\right)-\nabla \cdot\left(\varepsilon^{l} J_{\varepsilon}^{\mathrm{w}} \Psi_{\varepsilon}^{\mathrm{w}-1} \hat{D}_{\varepsilon}^{\mathrm{w}} \Psi_{\varepsilon}^{\mathrm{w}-T} \nabla \hat{u}_{\varepsilon}^{\mathrm{w}}\right)=-r_{\varepsilon}^{\mathrm{u}} \hat{f}_{\varepsilon}^{\mathrm{r}}, \quad x \in \Omega_{\varepsilon}^{\mathrm{w}}, t \in S, \quad(3 \mathrm{a}) \\
\partial_{t}\left(J_{\varepsilon}^{\mathrm{w}} \hat{v}_{\varepsilon}^{\mathrm{w}}\right)-\nabla \cdot\left(\varepsilon^{l} J_{\varepsilon}^{\mathrm{w}} \Psi_{\varepsilon}^{\mathrm{w}-1} \hat{E}_{\varepsilon}^{\mathrm{w}} \Psi_{\varepsilon}^{\mathrm{w}-T} \nabla \hat{v}_{\varepsilon}^{\mathrm{w}}\right)=+r_{\varepsilon}^{\mathrm{v}} \hat{f}_{\varepsilon}^{\mathrm{r}}, \quad x \in \Omega_{\varepsilon}^{\mathrm{w}}, t \in S, \\
-\left(J_{\varepsilon}^{\mathrm{a}} \Psi_{\varepsilon}^{\mathrm{a}-1} \hat{D}_{\varepsilon}^{\mathrm{a}} \Psi_{\varepsilon}^{\mathrm{a}-T} \nabla \hat{u}_{\varepsilon}^{\mathrm{a}}\right) \cdot \nu_{\varepsilon}^{\mathrm{a}}=\left(\varepsilon^{l} J_{\varepsilon}^{\mathrm{w}} \Psi_{\varepsilon}^{\mathrm{w}-1} \hat{D}_{\varepsilon}^{\mathrm{w}} \Psi_{\varepsilon}^{\mathrm{w}-T} \nabla \hat{u}_{\varepsilon}^{\mathrm{w}}\right) \cdot \nu_{\varepsilon}^{\mathrm{w}}, & x \in \partial \Omega_{\varepsilon}^{\mathrm{a}} \cap \partial \Omega_{\varepsilon}^{\mathrm{w}}, t \in S, \\
-\left(J_{\varepsilon}^{\mathrm{a}} \Psi_{\varepsilon}^{\mathrm{a}-1} \hat{D}_{\varepsilon}^{\mathrm{a}} \Psi_{\varepsilon}^{\mathrm{a}-T} \nabla \hat{u}_{\varepsilon}^{\mathrm{a}}\right) \cdot \nu_{\varepsilon}^{\mathrm{a}}=\varepsilon \hat{a}_{\varepsilon}\left\|\Psi_{\varepsilon}^{\mathrm{w}-T} \nu_{\varepsilon}^{\mathrm{w}}\right\| J_{\varepsilon}^{\mathrm{w}}\left(\hat{u}_{\varepsilon}^{\mathrm{a}}-\hat{u}_{\varepsilon}^{\mathrm{w}}\right), & x \in \partial \Omega_{\varepsilon}^{\mathrm{a}} \cap \partial \Omega_{\varepsilon}^{\mathrm{w}}, t \in S, \\
-\left(J_{\varepsilon}^{\mathrm{a}} \Psi_{\varepsilon}^{\mathrm{a}-1} \hat{D}_{\varepsilon}^{\mathrm{a}} \Psi_{\varepsilon}^{\mathrm{a}-T} \nabla \hat{u}_{\varepsilon}^{\mathrm{a}}\right) \cdot \nu_{\varepsilon}^{\mathrm{a}}=0, & x \in \partial \Omega_{\varepsilon}^{\mathrm{a}} \cap \partial \Omega_{\varepsilon}^{\mathrm{s}}, t \in S, \\
-\left(\varepsilon^{l} J_{\varepsilon}^{\mathrm{w}} \Psi_{\varepsilon}^{\mathrm{w}-1} \hat{D}_{\varepsilon}^{\mathrm{w}} \Psi_{\varepsilon}^{\mathrm{w}-T} \nabla \hat{u}_{\varepsilon}^{\mathrm{w}}\right) \cdot \nu_{\varepsilon}^{\mathrm{w}}=0, & x \in \partial \Omega_{\varepsilon}^{\mathrm{w}} \cap \partial \Omega_{\varepsilon}^{\mathrm{s}}, t \in S, \\
-\left(\varepsilon^{l} J_{\varepsilon}^{\mathrm{w}} \Psi_{\varepsilon}^{\mathrm{w}-1} \hat{E}_{\varepsilon}^{\mathrm{w}} \Psi_{\varepsilon}^{\mathrm{w}-T} \nabla \hat{v}_{\varepsilon}^{\mathrm{w}}\right) \cdot \nu_{\varepsilon}^{\mathrm{w}}=0, & x \in \partial \Omega_{\varepsilon}^{\mathrm{w}}, t \in S,
\end{array}
$$

where quantities with a hat in the reference configuration correspond to the same quantities without a hat in the current configuration, $\hat{f}_{\varepsilon}^{\mathrm{r}}(X, t)=f_{\varepsilon}^{\mathrm{r}}\left(\psi_{\varepsilon}^{\mathrm{w}}(X, t), t\right)$, e.g. The scaling factor $\varepsilon^{l}$, where $l \in[0,2]$, associated with $D_{\varepsilon}^{\mathrm{w}}$ and $E_{\varepsilon}^{\mathrm{w}}$, arises from a nondimensionalization. It is related to the characteristic macroscopic and microscopic length scales as well as the diffusion times. Similar considerations motivate the scaling of the interfacial-exchange coefficient $a_{\varepsilon}$. To be more general, it would be necessary to use different scaling exponents for $D_{\varepsilon}^{\mathrm{w}}$ and $E_{\varepsilon}^{\mathrm{w}}$ and to also scale $D_{\varepsilon}^{\mathrm{a}}$ and $a_{\varepsilon}$ with arbitrary powers of $\varepsilon$. It might also be of interest to consider more general nonlinear interfacial-exchange kinetics such as those considered in [6]. Since this is not the focus of this work, we retain this semi-general scaling and the linear interfacial-exchange term and we also refer to $[7,8]$ for more details on the influence and choice of different scaling exponents.

We assume $\Omega_{\varepsilon}^{\mathrm{a}}$ and, if $l=0, \Omega_{\varepsilon}^{\mathrm{w}}$ to be connected. Since the evolution of the domains is assumed to be induced by the reaction (1), the evolution mappings describing the evolution, $\psi_{\varepsilon}^{i}$, are not known beforehand, but are coupled to the reaction-diffusion process itself. This connection is discussed in the next subsection. 


\subsection{Modelling the functions related to the evolution of the domain}

The reaction (1) consumes a matrix constituent and produces another one. Therefore, we need to model the evolution of the subdomains depending on the reaction. Since the functions $\psi_{\varepsilon}^{i}$ do not appear in any of the equations, but only the functions $J_{\varepsilon}^{i}$ and $\Psi_{\varepsilon}^{i}, i \in\{\mathrm{a}, \mathrm{w}\}$, we only need to model these latter quantities. The exact evolution of all subdomains, i.e. the functions $\psi_{\varepsilon}^{i}$, $i \in\{\mathrm{a}, \mathrm{w}, \mathrm{s}\}$, are not required. For this, we note that $\Psi_{\varepsilon}^{i}$ relates the length and orientation of a material fibre in the reference configuration to its length and orientation in the current configuration and $J_{\varepsilon}^{i}$ describes the change of volume.

We consider the case where it is reasonable to assume that changes in geometry other than the volumetric changes do not have a large influence on the problem, i.e. we can assume $\Psi_{\varepsilon}^{i} \approx$ Id in (3). Since it is reasonable to assume the water incompressible, we have $J^{\mathrm{w}} \equiv 1$ for all times. In particular, this implies $\left|Z^{\mathrm{w}}(t)\right|=\left|Z^{\mathrm{w}}(0)\right|$. Therefore, we are left with deriving an equation for $J^{\mathrm{a}}$. We assume the dissolution of $\mathrm{B}$ and the precipitation of $\mathrm{C}$ to be instantaneous in order to keep things simple. Because we need to relate the change of pore-air volume to the reaction in the pore water, we only obtain a (non-local) relation of the average quantities. Since $\Omega$ is given as a union of the $\varepsilon$-periodic domains $\Omega_{\varepsilon}^{\mathrm{a}}, \Omega_{\varepsilon}^{\mathrm{w}}$ and $\Omega_{\varepsilon}^{\mathrm{s}}$, it is reasonable to consider the averages with respect to each scaled cell. It is worth noting that this averaging is an important step in enabling the analysis of the next section.

Considering one cell $Y$, the amount (i.e. mass) of constituent B being used up and of constituent $\mathrm{C}$ being produced at time $t$ is given by

$$
\int_{Z^{\mathrm{w}}(t)} m_{\mathrm{B}} \tilde{f}^{\mathrm{r}}(x, t) \mathrm{d} x \quad \text { and } \quad \int_{Z^{\mathrm{w}}(t)} m_{\mathrm{C}} \tilde{f}^{\mathrm{r}}(x, t) \mathrm{d} x
$$

respectively, where $\tilde{f}^{\mathrm{r}}$ is the production rate associated with the reaction of $\mathrm{B}$ and $\mathrm{C}$ in $Y$ and $m_{\mathrm{B}}$ and $m_{\mathrm{C}}$ are their molar weights. This corresponds to a change of volume of

$$
\left|Z^{\mathrm{a}}\right| C^{\mathrm{m}} \int_{Z^{\mathrm{w}}(t)} \tilde{f}^{\mathrm{r}}(x, t) \mathrm{d} x, \quad \text { where } C^{\mathrm{m}}=\frac{1}{\left|Z^{\mathrm{a}}\right|}\left(\frac{m_{\mathrm{C}}}{\rho_{\mathrm{C}}}-\frac{m_{\mathrm{B}}}{\rho_{\mathrm{B}}}\right)
$$

and $\rho_{\mathrm{B}}$ and $\rho_{\mathrm{C}}$ are the densities of constituents $\mathrm{B}$ and $\mathrm{C}$, respectively. On the other hand, the change of volume of the pore-air space is given by

$$
\frac{\mathrm{d}}{\mathrm{d} t} \int_{Z^{\mathrm{a}}(t)} 1 \mathrm{~d} x
$$

Noting that the an increase of total volume of constituents B and C implies a 
decrease of volume of pore air, we have

$$
\frac{\mathrm{d}}{\mathrm{d} t} \int_{Z^{\mathrm{a}}(t)} 1 \mathrm{~d} x=-\left|Z^{\mathrm{a}}\right| C^{\mathrm{m}} \int_{Z^{\mathrm{w}}(t)} \tilde{f}^{\mathrm{r}}(x, t) \mathrm{d} x
$$

for each instant in time. This can be transformed to the reference configuration and since $\tilde{J}^{\mathrm{w}} \equiv 1$, we obtain

$$
\int_{Z^{\mathrm{a}(0)}} \partial_{t} \tilde{J}^{\mathrm{a}}(X, t) \mathrm{d} X=-\left|Z^{\mathrm{a}}\right| C^{\mathrm{m}} \int_{Z^{\mathrm{w}}(0)} \hat{\tilde{f}}^{\mathrm{r}}(X, t) \mathrm{d} X, \quad J_{1}^{\mathrm{a}}(X, 0)=1 .
$$

$\tilde{J}^{\mathrm{a}}$ and $\hat{\tilde{f}}^{\mathrm{r}}$ are defined on different spatial domains (that is $Z^{\mathrm{a}}$ and $Z^{\mathrm{w}}$, respectively). Consequently, (8) only determines the average (or the total change) of $J^{\text {a }}$ with respect to each cell.

In order to obtain an equation in $\Omega$, we use the periodic unfolding operator $\mathcal{T}_{\varepsilon}[9]$ : writing $[x]_{Y}$ for the unique integer combination $\sum_{i=1}^{n} k_{i} e_{i}$ of the periods such that $\{x\}_{Y}=x-[y]_{Y}$ belongs to $[0,1)^{N}$ (where $e_{i}$ is the $i$ th unit vector), $\mathcal{T}_{\varepsilon} L^{2}(\Omega) \rightarrow L^{2}(\Omega \times Y)$ is defined by $T_{\varepsilon}(u)(x, y)=u\left(\varepsilon[x / \varepsilon]_{Y}+\varepsilon y\right)$. Thus, equation (8) can be written as

$$
\int_{Y} \chi^{\mathrm{a}}(y) \mathcal{T}_{\varepsilon}\left(\partial_{t} \tilde{J}^{\mathrm{a}}\right)(x, y, t) \mathrm{d} y=-\left|Z^{\mathrm{a}}\right| C^{\mathrm{m}} \int_{Y} \chi^{\mathrm{w}}(y) \mathcal{T}_{\varepsilon}\left(\hat{f}_{\varepsilon}^{\mathrm{r}}\right)(x, y, t) \mathrm{d} y
$$

for a.e. $x \in \Omega$. Since we do not expect $J^{\mathrm{a}}$ (the limit of $J_{\varepsilon}^{\mathrm{a}}$ as $\varepsilon \rightarrow 0$ ) to vary within one cell (and since we do not know any better), we define $J_{\varepsilon}^{\mathrm{a}}(x, t)=$ $\mathcal{T}_{\varepsilon}\left(\tilde{J}^{\mathrm{a}}\right)(x, t)$, which we assume constant with respect to $y$. Integration with respect to $x$ and using that $\partial_{t} J^{\mathrm{a}}$ is constant in each cell, we obtain

$$
\left|Z^{\mathrm{a}}\right| \int_{\Omega} \partial_{t} J_{\varepsilon}^{\mathrm{a}}(x, t) \mathrm{d} x=-\left|Z^{\mathrm{a}}\right| C^{\mathrm{m}} \int_{\Omega} \int_{Z^{\mathrm{w}}} \mathcal{T}_{\varepsilon}\left(\hat{f}_{\varepsilon}^{\mathrm{r}}\right)(x, y, t) \mathrm{d} y \mathrm{~d} x .
$$

Thus, the problem for $J_{\varepsilon}^{\mathrm{a}}$ is as follows: find $J_{\varepsilon}^{\mathrm{a}}: \Omega \times S \rightarrow \mathbb{R}$ such that

$$
\partial_{t} J_{\varepsilon}^{\mathrm{a}}(x, t)=-C^{\mathrm{m}} \bar{f}_{\varepsilon}^{\mathrm{r}}(x, t), \quad J_{\varepsilon}^{\mathrm{a}}(0) \equiv 1,
$$

where we have written

$$
\bar{f}_{\varepsilon}^{\mathrm{r}}(x, t)=\int_{Z^{\mathrm{w}}} \mathcal{T}_{\varepsilon}\left(\hat{f}_{\varepsilon}^{\mathrm{r}}\right)(x, y, t) \mathrm{d} y
$$

for ease of notation.

\subsection{Some remarks about the assumptions and on previous works}

In the derivation of (11), it was assumed that the change of volume of the solid matrix occurs at the same (macroscopic) place where the dissolution 
of constituent $\mathrm{B}$ and the precipitation (and attachment) of the product $\mathrm{C}$ happens. In other words, dissolution, reaction and precipitation occur in the same macroscopic place. Using the above approach, it would also be possible to derive an equation for $J^{\text {a }}$ accounting for these processes occurring in different places. Then, the right-hand side of the balance equation (7) would have to incorporate the amount of dissolved and precipitated constituent in the cell under consideration (as opposed to the production rate by reaction).

Furthermore, this approach neglects possible fluxes due to the change of volume. For example, if the solid matrix grows in one place, the air might be pushed away and, in turn, carry away gaseous reactants in it. Such a transport is neglected in this approach.

Similar macroscopic equations for the evolution of the porosity can be found in the literature. Most similar are the equations derived by Logan [10]. He considers a related problem where reactions in mineral rocks cause a change to the volume fraction of the solid matrix. Also, Muntean [11] derived an equation for the change of the porosity in concrete using very similar arguments. However, he starts from the assumption that the porosity does not depend on the spatial variable and, in turn, he obtains an equation which can be considered as an (macroscopically) averaged version of (11). It is important to note that both authors model the evolution of the porosity from a purely macroscopic point of view. Consequently, they do not come by equations of the type of (11).

An equation for the porosity incorporating microscopic effects was derived by Wilmański [12]. However, these considerations are rather abstract and therefore, the terms appearing in the balance equation (cf. eq. (10.51) in [12]), namely macroscopical and microscopical velocities of the matrix skeleton, are usually unknown and need to be related to the other unknowns by additional constitutive relations or assumptions. Nevertheless, it is worth noting that his balance equation is of parabolic type where the elliptic part incorporates the (macroscopic) bulk velocity of the skeleton. In subsequent chapters, he adjusts (simplifies, that is) the equation for specific problems and arrives at similar observations as the ones obtained in the above derivation. However, changes due to chemical reactions are not considered in [12] and, consequently, an equation comparable to (11) is not obtained.

It was stated previously that we want to concentrate on the evolution of $J_{\varepsilon}^{\mathrm{a}}$ and assume $\Psi_{\varepsilon}^{\mathrm{a}}=\Psi_{\varepsilon}^{\mathrm{w}}=\mathrm{Id}$. Of course, this is a rather strong assumption. Nevertheless, the resulting limit systems of equations are of a form which is often found in the engineering literature, namely the effective diffusivity is a product of the molecular diffusivity (the microscopic diffusivity in our terminology), a fixed factor accounting for the cell geometry (this corresponds to the tensor $P^{\mathrm{a}}$ calculated with unit diffusivity; it is sometimes called tortuosity) and an 
evolving volume factor which directly corresponds to $J^{\text {a }}$, cf. $[13,14]$, for example. Moreover, the resulting type of macroscopic system of equations yields good agreement with experimental data in the case of concrete carbonation [8].

It is noteworthy that the results for a simplified version of the system considered here have recently been published in a short note [15]. There, the reaction-diffusion system consists of only two equations but the evolution of $J_{\varepsilon}^{\mathrm{a}}$ is modelled in the same way as it is here. The rigorous proofs missing in the short note can be obtained in exact analogy to those presented here.

\subsection{Outline of what follows}

In the next section, the reaction-diffusion system under consideration is stated concisely in strong and weak form and the assumptions on the data are given. The macroscopic system obtained in the homogenization limit as $\varepsilon \rightarrow 0$ is stated in $\S 3$. The subsequent sections are concerned with rigorously proving these results: Existence of solutions of the microscopic system, $\varepsilon$-independent a-priori estimates and the convergence of the sequences of micro-solutions are shown in $\S 4$. Using these results, $\S 5$ is concerned with the identification of the homogenized limit problems summarized in $\S 3$.

\section{The reaction-diffusion system}

We want to investigate the reaction-diffusion problem with evolving microstructure, i.e. problem (3), where the evolution of the air-filled volume is determined by reaction as is modelled by (11). Recall that we have $J_{\varepsilon}^{\mathrm{w}} \equiv 1$. We assume $\partial \Omega_{\varepsilon}^{\mathrm{a}} \cap \partial \Omega_{\varepsilon}^{\mathrm{s}}=\emptyset$ for simplicity. Note that this does not induce any restriction from a modelling point of view since nothing happens across this boundary. Moreover, we write $\Gamma_{\varepsilon}=\partial \Omega_{\varepsilon}^{\mathrm{a}} \cap \partial \Omega_{\varepsilon}^{\mathrm{w}}, \Gamma_{\varepsilon}^{\mathrm{ws}}=\partial \Omega_{\varepsilon}^{\mathrm{w}} \cap \partial \Omega_{\varepsilon}^{\mathrm{s}}$ and $\Gamma_{\varepsilon}^{\mathrm{w}}=\partial \Omega_{\varepsilon}^{\mathrm{w}} \backslash \partial \Omega$.

Dropping the hats for notational convenience, the reaction-diffusion problem under consideration is thus given by

$$
\begin{aligned}
\partial_{t}\left(J_{\varepsilon}^{\mathrm{a}} u_{\varepsilon}^{\mathrm{a}}(x, t)\right)-\nabla \cdot\left(J_{\varepsilon}^{\mathrm{a}} D_{\varepsilon}^{\mathrm{a}} \nabla u_{\varepsilon}^{\mathrm{a}}\right) & =0, & & x \in \Omega_{\varepsilon}^{\mathrm{a}}, t \in S, \\
\partial_{t} u_{\varepsilon}^{\mathrm{w}}(x, t)-\nabla \cdot\left(\varepsilon^{l} D_{\varepsilon}^{\mathrm{w}} \nabla u_{\varepsilon}^{\mathrm{w}}\right) & =-r_{\varepsilon}^{\mathrm{u}} f_{\varepsilon}^{\mathrm{r}}, & & x \in \Omega_{\varepsilon}^{\mathrm{w}}, t \in S, \\
\partial_{t} v_{\varepsilon}^{\mathrm{w}}(x, t)-\nabla \cdot\left(\varepsilon^{l} E_{\varepsilon}^{\mathrm{w}} \nabla v_{\varepsilon}^{\mathrm{w}}\right) & =+r_{\varepsilon}^{\mathrm{v}} f_{\varepsilon}^{\mathrm{r}}, & & x \in \Omega_{\varepsilon}^{\mathrm{w}}, t \in S,
\end{aligned}
$$




$$
\begin{aligned}
& -\left(J_{\varepsilon}^{\mathrm{a}} D_{\varepsilon}^{\mathrm{a}} \nabla u_{\varepsilon}^{\mathrm{a}}\right) \cdot \nu_{\varepsilon}^{\mathrm{a}}=\left(\varepsilon^{l} D_{\varepsilon}^{\mathrm{w}} \nabla u_{\varepsilon}^{\mathrm{w}}\right) \cdot \nu_{\varepsilon}^{\mathrm{w}}, \quad x \in \Gamma_{\varepsilon}, t \in S, \\
& -\left(J_{\varepsilon}^{\mathrm{a}} D_{\varepsilon}^{\mathrm{a}} \nabla u_{\varepsilon}^{\mathrm{a}}\right) \cdot \nu_{\varepsilon}^{\mathrm{a}}=\varepsilon a_{\varepsilon}\left(u_{\varepsilon}^{\mathrm{a}}-u_{\varepsilon}^{\mathrm{w}}\right), \quad x \in \Gamma_{\varepsilon}, t \in S, \\
& -\left(\varepsilon^{l} D_{\varepsilon}^{\mathrm{w}} \nabla u_{\varepsilon}^{\mathrm{w}}\right) \cdot \nu_{\varepsilon}^{\mathrm{w}}=0, \quad x \in \Gamma_{\varepsilon}^{\mathrm{ws}}, t \in S, \\
& -\left(\varepsilon^{l} E_{\varepsilon}^{\mathrm{w}} \nabla v_{\varepsilon}^{\mathrm{w}}\right) \cdot \nu_{\varepsilon}^{\mathrm{w}}=0, \quad x \in \Gamma_{\varepsilon}^{\mathrm{w}}, t \in S,
\end{aligned}
$$

subject to homogeneous Neumann conditions at the exterior boundary $\partial \Omega$ and initial conditions and we assume that the evolution of the porosity factor $J_{\varepsilon}^{\text {a }}$ can be described by an equation of the form (11),

$$
\partial_{t} J_{\varepsilon}^{\mathrm{a}}(x, t)=-C^{\mathrm{m}} \bar{f}_{\varepsilon}^{\mathrm{r}}, \quad J_{\varepsilon}^{\mathrm{a}}(0) \equiv 1,
$$

where we have used the notation introduced in (12).

Let $\mathcal{V}(\Omega)=L^{2}\left(0, T ; W^{1,2}(\Omega)\right)$

$$
(u(t) \mid v(t))_{\Omega}=\int_{\Omega} u(x, t) v(x, t) \mathrm{d} x, \quad(u \mid v)_{\Omega, t}=\int_{0}^{t}(u(t) \mid v(t))_{\Omega} \mathrm{d} t
$$

$|u(t)|_{\Omega}^{2}=(u(t) \mid u(t))_{\Omega}$ and $|u|_{\Omega, t}^{2}=(u \mid u)_{\Omega, t}$. For the coefficients of the system, we assume that their space variable can be split into a macroscopic and a microscopic one (often called slow and fast component): For $\alpha \in\{\mathrm{a}, \mathrm{w}\}$, we assume that there exist functions $D^{\alpha}=D^{\alpha}(x, y, t), x \in \Omega_{\varepsilon}^{\alpha}, y \in Z^{\alpha}, t \in S$, bounded from above and from below by numbers $D_{\max }^{\alpha}$ and $D_{\min }^{\alpha}$, respectively, and periodically extended in $y$, such that $D_{\varepsilon}^{\alpha}(x, t)=D^{\alpha}(x, x / \varepsilon, t)$. We further assume

$$
\lim _{\varepsilon \rightarrow 0}\left|D_{\varepsilon}^{\alpha}(t)\right|_{\Omega}^{2}=\left|D^{\alpha}(t)\right|_{\Omega \times Y}^{2}
$$

and analogously for $E_{\varepsilon}^{\mathrm{w}}, a_{\varepsilon}, r_{\varepsilon}^{\mathrm{u}}$ and $r_{\varepsilon}^{\mathrm{v}}$. These function are extended to all of $\Omega$ by zero. Furthermore, we assume $r_{\min }^{\mathrm{u}}, r_{\min }^{\mathrm{v}} \geq 0$ and $D_{\min }^{\alpha}, E_{\min }^{\mathrm{w}}, a_{\min }>0$ and that

$$
\lim _{\varepsilon \rightarrow 0} \varepsilon\left|a_{\varepsilon}(t)\right|_{\Gamma_{\varepsilon}}^{2}=|a(t)|_{\Omega \times \Gamma}^{2}
$$

holds for $a$ instead of (15). We also require $\partial_{t} D_{\varepsilon}^{\alpha}, \partial_{t} E_{\varepsilon}^{\mathrm{w}}, \partial_{t} a_{\varepsilon}$ bounded from above.

We assume that the function $f_{\varepsilon}^{\mathrm{r}}(x, t)$ is given and bounded independently of $\varepsilon$ in $L^{2}\left(\Omega_{\varepsilon}^{\mathrm{w}} \times S\right)$. For some results, it will turn out that we moreover need $f_{\varepsilon}^{\mathrm{r}}$ to be bounded in $L^{\infty}\left(S ; L^{2}\left(\Omega_{\varepsilon}^{\mathrm{w}}\right)\right)$ if $C^{\mathrm{m}}>0$.

Therefore, the complete weak form of the problem under consideration reads as follows: Given $\varepsilon>0, C^{\mathrm{m}} \in \mathbb{R}$ and $f_{\varepsilon}^{\mathrm{r}} \in L^{2}\left(\Omega_{\varepsilon}^{\mathrm{w}} \times S\right)$, find $\left(u_{\varepsilon}^{\mathrm{a}}, u_{\varepsilon}^{\mathrm{w}}, v_{\varepsilon}^{\mathrm{w}}\right) \in$ $\mathcal{V}\left(\Omega_{\varepsilon}^{\mathrm{a}}\right) \times\left[\mathcal{V}\left(\Omega_{\varepsilon}^{\mathrm{w}}\right)\right]^{2}$ and $J_{\varepsilon}^{\mathrm{a}} \in W^{1,2}\left(S ; L^{2}(\Omega)\right)$ such that $\left(u_{\varepsilon}^{\mathrm{a}}(0), u_{\varepsilon}^{\mathrm{w}}(0), v_{\varepsilon}^{\mathrm{w}}(0)\right)=$ 


$$
\begin{gathered}
\left(u_{0}^{\mathrm{a}}, u_{0}^{\mathrm{w}}, v_{0}^{\mathrm{w}}\right) \in\left[L^{2}(\Omega)\right]^{3}, J_{\varepsilon}^{\mathrm{a}}(0)=1 \text { and } \\
\left(\partial_{t} J_{\varepsilon}^{\mathrm{a}}(t) \mid \varphi^{\mathrm{J}}(t)\right)_{\Omega}=-C^{\mathrm{m}}\left(\bar{f}_{\varepsilon}^{\mathrm{r}}(t) \mid \varphi^{\mathrm{J}}(t)\right)_{\Omega} \\
\left(\partial_{t}\left(J_{\varepsilon}^{\mathrm{a}}(t) u_{\varepsilon}^{\mathrm{a}}(t)\right) \mid \phi^{\mathrm{a}}(t)\right)_{\Omega_{\varepsilon}^{\mathrm{a}}}+\left(J_{\varepsilon}^{\mathrm{a}}(t) D_{\varepsilon}^{\mathrm{a}} \nabla u_{\varepsilon}^{\mathrm{a}}(t) \mid \nabla \phi^{\mathrm{a}}(t)\right)_{\Omega_{\varepsilon}^{\mathrm{a}}} \\
=-\varepsilon\left(a_{\varepsilon}(t)\left(u_{\varepsilon}^{\mathrm{a}}(t)-u_{\varepsilon}^{\mathrm{w}}(t)\right) \mid \phi^{\mathrm{a}}(t)\right)_{\Gamma_{\varepsilon}} \\
\left(\partial_{t} u_{\varepsilon}^{\mathrm{w}}(t) \mid \phi^{\mathrm{w}}(t)\right)_{\Omega_{\varepsilon}^{\mathrm{w}}}+\varepsilon^{l}\left(D_{\varepsilon}^{\mathrm{w}} \nabla u_{\varepsilon}^{\mathrm{w}}(t) \mid \nabla \phi^{\mathrm{w}}(t)\right)_{\Omega_{\varepsilon}^{\mathrm{w}}} \\
=-\left(r_{\varepsilon}^{\mathrm{u}}(t) f_{\varepsilon}^{\mathrm{r}}(t) \mid \phi^{\mathrm{w}}(t)\right)_{\Omega_{\varepsilon}^{\mathrm{w}}}+\varepsilon\left(a_{\varepsilon}\left(u_{\varepsilon}^{\mathrm{a}}(t)-u_{\varepsilon}^{\mathrm{w}}(t)\right) \mid \phi^{\mathrm{w}}(t)\right)_{\Gamma_{\varepsilon}} \\
\left(\partial_{t} v_{\varepsilon}^{\mathrm{w}}(t) \mid \varphi^{\mathrm{w}}(t)\right)_{\Omega_{\varepsilon}^{\mathrm{w}}}+\varepsilon^{l}\left(E_{\varepsilon}^{\mathrm{w}}(t) \nabla v_{\varepsilon}^{\mathrm{w}}(t) \mid \nabla \varphi^{\mathrm{w}}(t)\right)_{\Omega_{\varepsilon}^{\mathrm{w}}} \\
=+\left(r_{\varepsilon}^{\mathrm{v}}(t) f_{\varepsilon}^{\mathrm{r}}(t) \mid \varphi^{\mathrm{w}}(t)\right)_{\Omega_{\varepsilon}^{\mathrm{w}}}
\end{gathered}
$$

for all $\left(\phi^{\mathrm{a}}, \phi^{\mathrm{w}}, \varphi^{\mathrm{w}}, \varphi^{\mathrm{J}}\right) \in \mathcal{V}\left(\Omega_{\varepsilon}^{\mathrm{a}}\right) \times\left[\mathcal{V}\left(\Omega_{\varepsilon}^{\mathrm{w}}\right)\right]^{2} \times L^{2}(\Omega \times S)$ and a.e. $t \in S$.

For the proof of existence of microsolutions, we make use of an abstract theory for degenerate problems, developed in [16], in $\S 2$. It is worth noting that the particular modelling of $J_{\varepsilon}^{\mathrm{a}}$, that is assuming it constant in each cell, permits the analysis carried out here as it allows to proof the boundedness of $J_{\varepsilon}^{\mathrm{a}}$ in almost every point of $\Omega$. This result is quite essential for the existence proofs. Moreover, the case that the pore-air volume shrinks is more difficult than the case of increasing pore-air volume. This is due to the fact that, for a constant amount of molecules in a given volume, the concentration increases for decreasing volume while the concentration decreases for increasing volume.

We begin the analysis with the investigation of the equation for $J_{\varepsilon}^{\mathrm{a}}$. Then, the existence of microsolutions is proven before showing a-priori estimates and discussing the convergence using the notions of two-scale convergence.

\section{The macroscopic limit problem}

We state the macroscopic limit problem of (13) as $\varepsilon \rightarrow 0$. We first define a generic cell problem: Let the functions $\varsigma_{j}, j=1, \ldots, N$, be the $Y$-periodic solution of the cell problem

$$
\begin{gathered}
-\nabla_{y} \cdot\left(A(x, y, t)\left(\nabla_{y} \varsigma_{j}(x, y, t)+e_{j}\right)\right)=0, \quad y \in Z^{\alpha}, x \in \Omega, t \in S, \\
-A(x, y, t)\left(\nabla_{y} \varsigma_{j}(x, y, t)+e_{j}\right) \cdot \nu^{\alpha}=0, \quad y \in \Gamma, x \in \Omega, t \in S,
\end{gathered}
$$

the weak form of which is given by

$$
\left(A(x, \cdot, t)\left(\nabla_{y} \varsigma_{j}(x, \cdot, t)+e_{j}\right) \mid \nabla_{y} \phi\right)_{Z^{\alpha}}=0
$$

for all $Y$-periodic test functions $\phi$. The vector $e_{j}$ is the $j$ th unit vector in $N$-dimensional Euclidean space. We will specify $A$ and $\alpha$ to our needs below. 
The limit problem corresponding to $J^{\text {a }}$ is given by

$$
\partial_{t} J^{\mathrm{a}}(x, t)=-C^{\mathrm{m}} \int_{Z^{\mathrm{w}}} f^{\mathrm{r}} \mathrm{d} y, \quad x \in \Omega, t \in S,
$$

with initial condition $J^{\mathrm{a}}(0)=1$. The limit equation for $u^{\mathrm{a}}$ states

$$
\partial_{t}\left(J^{\mathrm{a}} u^{\mathrm{a}}(x, t)\right)-\nabla \cdot\left(P^{\mathrm{a}} \nabla u^{\mathrm{a}}\right)=-\int_{\Gamma} a\left(u^{\mathrm{a}}-u^{\mathrm{w}}\right) \mathrm{d} \sigma_{y}, \quad x \in \Omega, t \in S,
$$

with homogeneous Neumann conditions at $\partial \Omega$ and initial condition $u^{\mathrm{a}}(0)=u_{0}^{\mathrm{a}}$, where $P^{\mathrm{a}}=\left[p_{i j}^{\mathrm{a}}\right]_{i j}$ is defined by

$$
p_{i j}^{\mathrm{a}}(x, t)=\int_{Z^{\mathrm{a}}} J^{\mathrm{a}}(x, t) D^{\mathrm{a}}(x, y, t)\left(\delta_{i j}+\partial_{y_{i}} \varsigma_{j}^{\mathrm{a}}(x, y, t)\right) \mathrm{d} y .
$$

The functions $\varsigma_{j}^{\mathrm{a}}, j=1, \ldots, N$, are the $Y$-periodic solutions of (18) with $A=J^{\mathrm{a}} D^{\mathrm{a}}$ and $\alpha=\mathrm{a}$ and $\delta_{i j}$ is the Kronecker delta.

The limit equations for $u^{\mathrm{w}}$ and $v^{\mathrm{w}}$ depend on the particular choice of the scaling exponent $l$. If $l<2$, the limit equations are given by

$\partial_{t} u^{\mathrm{w}}(x, t)-\delta_{0 l} \nabla \cdot\left(P^{\mathrm{w}} \nabla u^{\mathrm{w}}\right)=-\int_{Z^{\mathrm{w}}} r^{\mathrm{u}} f^{\mathrm{r}} \mathrm{d} y+\int_{\Gamma} a\left(u^{\mathrm{a}}-u^{\mathrm{w}}\right) \mathrm{d} \sigma_{y}, \quad x \in \Omega, t \in S$,

$\partial_{t} v^{\mathrm{w}}(x, t)-\delta_{0 l} \nabla \cdot\left(Q^{\mathrm{w}} \nabla v^{\mathrm{w}}\right)=+\int_{Z^{\mathrm{w}}} r^{\mathrm{v}} f^{\mathrm{r}} \mathrm{d} y$, $x \in \Omega, t \in S$,

where $P^{\mathrm{w}}=\left[p_{i j}^{\mathrm{w}}\right]_{i j}$ and $Q^{\mathrm{w}}=\left[q_{i j}^{\mathrm{w}}\right]_{i j}$ are defined by

$$
\begin{aligned}
& p_{i j}^{\mathrm{w}}(x, t)=\int_{Z^{\mathrm{w}}} D^{\mathrm{w}}(x, y, t)\left(\delta_{i j}+\partial_{y_{i}} \varsigma_{j}^{\mathrm{w}}(x, y, t)\right) \mathrm{d} y, \\
& q_{i j}^{\mathrm{w}}(x, t)=\int_{Z^{\mathrm{w}}} E^{\mathrm{w}}(x, y, t)\left(\delta_{i j}+\partial_{y_{i}} \sigma_{j}^{\mathrm{w}}(x, y, t)\right) \mathrm{d} y,
\end{aligned}
$$

where $\varsigma_{j}^{\mathrm{w}}$ and $\sigma_{j}^{\mathrm{w}}, j=1, \ldots, N$, are the $Y$-periodic solutions of (18) with $A=D^{\mathrm{w}}$ and $A=E^{\mathrm{w}}$, respectively, as well as $\alpha=\mathrm{w}$. If $l=2$, the limit equations are given by

$$
\begin{aligned}
& \partial_{t} u^{\mathrm{w}}(x, y, t)-\nabla_{y} \cdot\left(D^{\mathrm{w}} \nabla_{y} u^{\mathrm{w}}\right)=-r^{\mathrm{u}} f^{\mathrm{r}}, \quad x \in \Omega, y \in Z^{\mathrm{w}}, t \in S, \\
& \partial_{t} v^{\mathrm{w}}(x, y, t)-\nabla_{y} \cdot\left(E^{\mathrm{w}} \nabla_{y} v^{\mathrm{w}}\right)=+r^{\mathrm{v}} f^{\mathrm{r}}, \quad x \in \Omega, y \in Z^{\mathrm{w}}, t \in S, \\
& -D^{\mathrm{w}} \nabla_{y} u^{\mathrm{w}} \cdot \nu^{\mathrm{w}}=a\left(u^{\mathrm{a}}-u^{\mathrm{w}}\right), \quad x \in \Omega, y \in \Gamma, t \in S, \\
& -D^{\mathrm{w}} \nabla_{y} u^{\mathrm{w}} \cdot \nu^{\mathrm{w}}=0, \quad x \in \Omega, y \in \Gamma^{\mathrm{ws}}, t \in S \text {, } \\
& -E^{\mathrm{w}} \nabla_{y} v^{\mathrm{w}} \cdot \nu^{\mathrm{w}}=0, \quad x \in \Omega, y \in \Gamma^{\mathrm{w}}, t \in S .
\end{aligned}
$$


For all choices of $l$, homogeneous Neumann conditions apply at the exterior boundaries and $\left(u^{\mathrm{w}}(0), v^{\mathrm{w}}(0)\right)=\left(u_{0}^{\mathrm{w}}, u_{0}^{\mathrm{w}}\right)$.

\section{Existence, a-priori estimates, convergence}

\subsection{Existence and a-priori estimates for $J_{\varepsilon}^{\mathrm{a}}$}

Since the equation for $J_{\varepsilon}^{\mathrm{a}}$ is decoupled from the rest of the system, we can discuss it independently.

\section{Lemma 4.1}

For each $\varepsilon>0$, there exists a unique solution $J_{\varepsilon}^{\mathrm{a}}(x, t)$ of $(17 \mathrm{a})$ given by

$$
J_{\varepsilon}^{\mathrm{a}}(x, t)=1-C^{\mathrm{m}} \int_{0}^{t} \int_{Y} \mathcal{T}_{\varepsilon}\left(f_{\varepsilon}^{\mathrm{r}}\right)(x, y, s) \mathrm{d} y \mathrm{~d} s,
$$

which satisfies

$$
\left|J_{\varepsilon}^{\mathrm{a}}(t)\right|_{\Omega}^{2} \leq C, \quad\left|\partial_{t} J_{\varepsilon}^{\mathrm{a}}\right|_{\Omega, t}^{2} \leq C
$$

for a.e. $t \in S$. Moreover, $J_{\varepsilon}^{\mathrm{a}} \in L^{\infty}(\Omega \times S)$ and, if $f_{\varepsilon}^{\mathrm{r}} \in L^{p}\left(S ; L^{2}(\Omega)\right)$ for a $1 \leq p \leq \infty$, we have $\left|\partial_{t} J_{\varepsilon}^{\mathrm{a}}(x, t)\right| \leq K_{\varepsilon}(t)$ for a.e. $x \in \Omega$ and $t \in S$ with $K_{\varepsilon} \in L^{p}(S)$.

Proof The form of the solution (21), and, hence, its existence and uniqueness, follow by simple integration with respect to time.

Testing of $(17 \mathrm{a})$ with $J_{\varepsilon}^{\mathrm{a}}$ gives

$$
\frac{1}{2} \frac{\mathrm{d}}{\mathrm{d} t}\left|J_{\varepsilon}^{\mathrm{a}}(t)\right|_{\Omega}^{2}=-C^{\mathrm{m}}\left(\bar{f}_{\varepsilon}^{\mathrm{r}}(t) \mid J_{\varepsilon}^{\mathrm{a}}(t)\right)_{\Omega} .
$$

Application of the Cauchy-Schwarz and Young inequalities, integration with respect to time, and noting that $\left|\mathcal{T}_{\varepsilon}\left(f_{\varepsilon}^{\mathrm{r}}(t)\right)\right|_{\Omega \times Y}=\left|f_{\varepsilon}^{\mathrm{r}}(t)\right|_{\Omega}$ yields

$$
\left|J_{\varepsilon}^{\mathrm{a}}(t)\right|_{\Omega}^{2} \leq C^{\mathrm{m}}\left(\left|f_{\varepsilon}^{\mathrm{r}}\right|_{\Omega, t}^{2}+\left|J_{\varepsilon}^{\mathrm{a}}\right|_{\Omega, t}^{2}\right)+|\Omega|
$$

Application of Gronwall's inequality and using the assumptions of $f_{\varepsilon}^{\mathrm{r}}$ gives the first estimate in (22).

Testing with $\partial_{t} J_{\varepsilon}^{\mathrm{a}}$ gives

$$
\left|\partial_{t} J_{\varepsilon}^{\mathrm{a}}(t)\right|_{\Omega}^{2}=-C^{\mathrm{m}}\left(\bar{f}_{\varepsilon}^{\mathrm{r}}(t) \mid \partial_{t} J_{\varepsilon}^{\mathrm{a}}(t)\right)_{\Omega} .
$$

For $\delta>0$, we obtain

$$
\left|\partial_{t} J_{\varepsilon}^{\mathrm{a}}(t)\right|_{\Omega}^{2}=C\left(\delta^{-1}\left|f_{\varepsilon}^{\mathrm{r}}(t)\right|_{\Omega}^{2}+\delta\left|\partial_{t} J_{\varepsilon}^{\mathrm{a}}(t)\right|_{\Omega}^{2}\right) .
$$


Integration with respect to time and choosing $\delta$ small enough yields the second estimate in (22).

Moreover, for given $\varepsilon$ and $t, J_{\varepsilon}^{\mathrm{a}}(t)$ and $\partial_{t} J_{\varepsilon}^{\mathrm{a}}(t)$ take a finite number of values in $\Omega$. Since $J_{\varepsilon}^{\mathrm{a}}(t)$ is also continuous with respect to $t$, it belongs to $L^{\infty}(\Omega \times S)$. Furthermore,

$$
\left|\partial_{t} J_{\varepsilon}^{\mathrm{a}}(x, t)\right|=\left|C^{\mathrm{m}} \int_{Y} \mathcal{T}_{\varepsilon}\left(f_{\varepsilon}^{\mathrm{r}}\right)(x, y, t) \mathrm{d} y\right|=C^{\mathrm{m}} \int_{Y} \mathcal{T}_{\varepsilon}\left(f_{\varepsilon}^{\mathrm{r}}\right)(x, y, t) \mathrm{d} y \leq K_{\varepsilon}(t),
$$

where, defining $M_{\varepsilon}=\{$ midpoints of the cells in $\Omega$ (note that this set has a finite number of elements for given $\varepsilon>0$ ),

$$
K_{\varepsilon}(t)=C^{\mathrm{m}} \max _{x \in M_{\varepsilon}} \int_{Y} \mathcal{T}_{\varepsilon}\left(f_{\varepsilon}^{\mathrm{r}}\right)(x, y, t) \mathrm{d} y .
$$

It remains to be shown that $K_{\varepsilon} \in L^{p}(S)$. For $p=\infty$, this is clear. For $p<\infty$, note that the space $\left(\mathbb{R}^{\# M_{\varepsilon}},\|\cdot\|_{1}\right)$ is continuously embedded in $\left(\mathbb{R}^{\# M_{\varepsilon}},\|\cdot\|_{\max }\right)$. Denoting the embedding constant by $\tilde{C}_{\varepsilon}$ and noting that $f_{\varepsilon}^{\mathrm{r}}$ is non-negative, we obtain

$$
\begin{aligned}
\int_{0}^{T}\left(K_{\varepsilon}(t)\right)^{p} \mathrm{~d} t & =\left(C^{\mathrm{m}}\right)^{p}\left(\int_{0}^{T} \max _{x \in M_{\varepsilon}} \int_{Y} \mathcal{T}_{\varepsilon}\left(f_{\varepsilon}^{\mathrm{r}}\right)(x, y, t) \mathrm{d} y \mathrm{~d} t\right)^{p} \\
& \leq\left(C^{\mathrm{m}}\right)^{p} T^{\frac{p}{p^{\prime}}} \int_{0}^{T}\left(\max _{x \in M_{\varepsilon}} \int_{Y} \mathcal{T}_{\varepsilon}\left(f_{\varepsilon}^{\mathrm{r}}\right)(x, y, t) \mathrm{d} y\right)^{p} \mathrm{~d} t \\
& \leq\left(C^{\mathrm{m}}\right)^{p} T^{\frac{p}{p^{\prime}}}\left(\# M_{\varepsilon}\right)^{\frac{p}{p^{\prime}}} \tilde{C}_{\varepsilon}^{p} \int_{0}^{T} \sum_{x \in M_{\varepsilon}}\left(\int_{Y} \mathcal{T}_{\varepsilon}\left(f_{\varepsilon}^{\mathrm{r}}\right)(x, y, t) \mathrm{d} y\right)^{p} \mathrm{~d} t \\
& \leq\left(C^{\mathrm{m}}\right)^{p} T^{\frac{p}{p^{\prime}}}\left(\# M_{\varepsilon}\right)^{\frac{p}{p^{\prime}}} \tilde{C}_{\varepsilon}^{p} \sum_{x \in M_{\varepsilon}} \int_{Y} \mathcal{T}_{\varepsilon}\left(\int_{0}^{T}\left(f_{\varepsilon}^{\mathrm{r}}(\cdot, t)\right)^{p} \mathrm{~d} t\right)(x, y) \mathrm{d} y<\infty
\end{aligned}
$$

where we have made extensive use of Hölder's inequality and the boundedness follows from the fact that $f_{\varepsilon}^{\mathrm{r}}(x, \cdot) \in L^{p}(S)$ for a.e. $x$. Note that, in general, the $L^{\infty}$-norms depend on $\varepsilon$ and they may not be bounded for $\varepsilon \rightarrow 0$.

If $C^{\mathrm{m}}>0$, it is clear from (21) that the maximum time of applicability of this model is such that $J_{\varepsilon}^{\mathrm{a}}$ does not become negative. The model could be altered such that $J_{\varepsilon}^{\text {a }}$ remains zero after it has reached zero. We do not want to follow this direction here. Instead, we demand that $T$ is chosen small enough to allow $J_{\varepsilon}^{\mathrm{a}}(x, t) \geq J_{\min }^{\mathrm{a}}$ for a.e. $x \in \Omega$ and $t \in S$ for some $J_{\min }^{\mathrm{a}}>0$ independent of $\varepsilon$. This is equivalent to

$$
\int_{0}^{t} \int_{Y} \mathcal{T}_{\varepsilon}\left(f_{\varepsilon}^{\mathrm{r}}\right)(x, y, s) \mathrm{d} y \mathrm{~d} s \leq \frac{1-J_{\min }^{\mathrm{a}}}{C^{\mathrm{m}}}, \quad t \in(0, T) .
$$

Of course, this maximum time depends on the availability of the reactants and may never be reached. For example, if not enough reactant is initially available and no new reactant enters the sample, such clogging of the pores cannot occur. 
We want to show existence of solutions of the full problem (17) for given $\varepsilon>0$. Note that the existence of $J_{\varepsilon}^{\mathrm{a}}$ satisfying (17a) is already ensured by lemma 4.1. Since we want to use the abstract theory contained in [16], an operator formulation of $(17 \mathrm{~b})-(17 \mathrm{~d})$ is necessary. It is worth noting that this abstract theory has already been used to prove existence of solutions of a microscopic system of equations, which was then homogenized, in [17]. In their considerations, the coefficient in the time-derivative term was only space dependent, however. First of all, we recall the results of [16].

\section{Definition 4.2}

Let $W$ be a separable Hilbert space. The family of operators $\{\mathcal{B}(t) \mid t \in S\}$ with $\mathcal{B}(t) \in \mathcal{L}\left(W, W^{\prime}\right)$ for each $t \in S$ and $\mathcal{B}(\cdot) u(v) \in L^{\infty}(S)$ for each pair $u, v \in W$ is called regular if for each pair $u, v \in W$, the function $\mathcal{B}(\cdot) u(v)$ is absolutely continuous on $\bar{S}$ and there is a $K \in L^{1}(S)$ such that

$$
\left|\frac{\mathrm{d}}{\mathrm{d} t} \mathcal{B}(t) u(v)\right| \leq K(t)\|u\|\|v\|, \quad u, v \in W, \text { for a.e. } t \in S .
$$

\section{Theorem 4.3}

Let the separable Hilbert spaces $V$ and $\mathcal{V}=L^{2}(S ; V)$ with duals $V^{\prime}$ and $\mathcal{V}^{\prime}=$ $L^{2}\left(S ; V^{\prime}\right)$ be given. Suppose that $W$ is a Hilbert space containing $V$ and the injection $V \hookrightarrow W$ is continuous with $V$ dense in $W$. Assume that for each $t \in \bar{S}$, we are given an operator $\mathcal{A}(t) \in \mathcal{L}\left(V, V^{\prime}\right)$ such that $\mathcal{A}(\cdot) u(v) \in L^{\infty}(S)$ for each pair $u, v \in V$. Likewise, we are given a regular family of self-adjoint operators $\mathcal{B}(t) \in \mathcal{L}\left(W, W^{\prime}\right)$ with $\mathcal{B}(\cdot) u(v) \in L^{\infty}(S)$ for each pair $u, v \in W$. Furthermore, suppose that $\mathcal{B}(0)$ is monotone and there are numbers $\kappa, \lambda>0$ such that

$$
2 \mathcal{A}(t) v(v)+\lambda \mathcal{B}(t) v(v)+\mu \mathcal{B}^{\prime}(t) v(v) \geq \kappa\|v\|^{2}, \quad v \in V, 0 \leq t \leq T,
$$

is satisfied with $\mu=1$. Then, for given $u_{0} \in W$ and $f \in \mathcal{V}^{\prime}$, the problem

$$
u \in \mathcal{V}: \frac{\mathrm{d}}{\mathrm{d} t}(\mathcal{B}(t) u(t))+\mathcal{A}(t) u(t)=f(t) \text { in } \mathcal{V}^{\prime}, \quad(\mathcal{B} u)(0)=\mathcal{B}(0) u_{0},
$$

possesses at least one solution which satisfies

$$
\|u\|_{\mathcal{V}} \leq C(\kappa, \lambda)\left(\|f\|_{\mathcal{V}^{\prime}}^{2}+\mathcal{B}(0) u_{0}\left(u_{0}\right)\right)^{\frac{1}{2}} .
$$

If, in addition, each $\mathcal{B}(t)$ is monotone, $\mathcal{A}(t)$ is a regular family of self-adjoint operators and (25) is also satisfied with $\mu=0$, then the solution is unique.

Proof The existence follows from proposition 3.2 while the uniqueness is given by proposition 3.3 in chapter III.3 of [16]. 
In order to use theorem 4.3 for problem $(17 b)-(17 d)$, we define the following operators.

We set $V(\Omega)=W^{1,2}(\Omega), W(\Omega)=L^{2}(\Omega)$ and $V=V\left(\Omega_{\varepsilon}^{\mathrm{a}}\right) \times\left[V\left(\Omega_{\varepsilon}^{\mathrm{w}}\right)\right]^{2}$ and $W=$ $W\left(\Omega_{\varepsilon}^{\mathrm{a}}\right) \times\left[W\left(\Omega_{\varepsilon}^{\mathrm{w}}\right)\right]^{2}$. For each $t \in \bar{S}$ and $u=\left(u_{1}, u_{2}, u_{3}\right), v=\left(v_{1}, v_{2}, v_{3}\right) \in V$, define $\mathcal{A}_{\varepsilon}(t): V \rightarrow V^{\prime}$ by

$$
\begin{aligned}
\left(\mathcal{A}_{\varepsilon}(t) u\right)(v)= & \int_{\Omega_{\varepsilon}^{\mathrm{a}}} J_{\varepsilon}^{\mathrm{a}}(x, t) D_{\varepsilon}^{\mathrm{a}}(x, t) \nabla u_{1}(x) \nabla v_{1}(x) \mathrm{d} x \\
& +\varepsilon^{l} \int_{\Omega_{\varepsilon}^{\mathrm{w}}} D_{\varepsilon}^{\mathrm{w}}(x, t) \nabla u_{2}(x) \nabla v_{2}(x) \mathrm{d} x \\
& +\varepsilon^{l} \int_{\Omega_{\varepsilon}^{\mathrm{w}}} E_{\varepsilon}^{\mathrm{w}}(x, t) \nabla u_{3}(x) \nabla v_{3}(x) \mathrm{d} x \\
& +\varepsilon \int_{\Gamma_{\varepsilon}} a_{\varepsilon}(x, t)\left(u_{1}(x)-u_{2}(x)\right)\left(v_{1}(x)-v_{2}(x)\right) \mathrm{d} \sigma_{x}
\end{aligned}
$$

as well as $f_{\varepsilon}: \mathcal{V} \rightarrow \mathbb{R}$ by

$$
\begin{aligned}
f_{\varepsilon}(v)= & -\int_{0}^{T} \int_{\Omega_{\varepsilon}^{\mathrm{w}}} r_{\varepsilon}^{\mathrm{u}}(x, s) f_{\varepsilon}^{\mathrm{r}}(x, s) v_{2}(x, s) \mathrm{d} x \mathrm{~d} t \\
& -\int_{0}^{T} \int_{\Omega_{\varepsilon}^{\mathrm{w}}} r_{\varepsilon}^{\mathrm{v}}(x, s) f_{\varepsilon}^{\mathrm{r}}(x, s) v_{3}(x, s) \mathrm{d} x \mathrm{~d} t
\end{aligned}
$$

for $\in \mathcal{V}$. For $u=\left(u_{1}, u_{2}, u_{3}\right), v=\left(v_{1}, v_{2}, v_{3}\right) \in W$, define $\mathcal{B}_{\varepsilon}(t): W \rightarrow W^{\prime}$ by $\left(\mathcal{B}_{\varepsilon}(t) u\right)(v)=\int_{\Omega_{\varepsilon}^{\mathrm{a}}} J_{\varepsilon}^{\mathrm{a}}(x, t) u_{1}(x) v_{1}(x) \mathrm{d} x+\int_{\Omega_{\varepsilon}^{\mathrm{w}}} u_{2}(x) v_{2}(x) \mathrm{d} x+\int_{\Omega_{\varepsilon}^{\mathrm{w}}} u_{3}(x) v_{3}(x) \mathrm{d} x$.

It is clear that $f_{\varepsilon} \in \mathcal{V}^{\prime}$ and for each $t \in \bar{S}, \mathcal{A}_{\varepsilon}(t) \in \mathcal{L}\left(V, V^{\prime}\right), \mathcal{B}_{\varepsilon}(t) \in \mathcal{L}\left(W, W^{\prime}\right)$. Furthermore, problem (17b)-(17d) is equivalent to

$$
u \in \mathcal{V}: \frac{\mathrm{d}}{\mathrm{d} t}\left(\mathcal{B}_{\varepsilon}(t) u(t)\right)+\mathcal{A}_{\varepsilon}(t) u(t)=f_{\varepsilon}(t) \text { in } \mathcal{V}^{\prime}, \quad\left(\mathcal{B}_{\varepsilon} u\right)(0)=\mathcal{B}_{\varepsilon}(0) u_{0}
$$

In order to obtain existence of solutions by theorem 4.3, we remark that we have $\mathcal{A}_{\varepsilon}(\cdot) u(v) \in L^{\infty}(S)$ for each pair $u, v \in V$ and $\mathcal{B}_{\varepsilon}(\cdot) u(v) \in L^{\infty}(S)$ for each pair $u, v \in W$ by the regularity assumptions on $D_{\varepsilon}^{\mathrm{a}}, D_{\varepsilon}^{\mathrm{w}}, E_{\varepsilon}^{\mathrm{w}}$ and $a_{\varepsilon}$ and what we know about $J_{\varepsilon}^{\mathrm{a}}$ from lemma 4.1. For the existence of solutions, it remains to be shown that the $\mathcal{B}_{\varepsilon}(t)$ form a regular family of self-adjoint operators with $\mathcal{B}_{\varepsilon}(0)$ monotone and that $(25)$ is satisfied with $\mu=1$. For uniqueness, we further need to show that the $\mathcal{A}_{\varepsilon}(t)$ form a regular family of self-adjoint operators, that the $\mathcal{B}_{\varepsilon}(t)$ are monotone and that $(25)$ is satisfied with $\mu=0$.

\section{Lemma 4.4}

The families of operators $\mathcal{A}_{\varepsilon}(t)$ (defined by (28)) and $\mathcal{B}_{\varepsilon}(t)$ (defined by (30)) form regular families of self-adjoint operators in $V$ and $W$, respectively. Moreover, $\mathcal{B}_{\varepsilon}(t)$ and $\mathcal{B}_{\varepsilon}(0)$ are monotone. 
Proof It is clear from the structure of $\mathcal{A}_{\varepsilon}(t)$ and $\mathcal{B}_{\varepsilon}(t)$ that these operators are self-adjoint for each $t$. The $\mathcal{B}_{\varepsilon}(t)$ are monotone owing to the linearity and the non-negativity of $J_{\varepsilon}^{\mathrm{a}}$. In particular, $J_{\varepsilon}^{\mathrm{a}}(x, 0)=1$, so $\mathcal{B}_{\varepsilon}(0)=\mathrm{Id}$ which is clearly monotone.

We proceed by proving that the $\mathcal{B}_{\varepsilon}(t)$ form a regular family of operators in $W$ (cf. definition 4.2). Let $u, v \in W$. Since $J_{\varepsilon}^{\mathrm{a}}(x, \cdot)$ is in $W^{1,2}(S)$ for a.e. $x$ and $W^{1,2}(S) \hookrightarrow W^{1,1}(S) \cong A C(\bar{S}), \mathcal{B}_{\varepsilon}(\cdot) u(v)$ is absolutely continuous. Moreover,

$$
\begin{aligned}
\left|\frac{\mathrm{d}}{\mathrm{d} t} \mathcal{B}_{\varepsilon}(t) u(v)\right| & =\left|\int_{\Omega_{\varepsilon}^{\mathrm{a}}} \partial_{t} J_{\varepsilon}^{\mathrm{a}}(x, t) u_{1}(x) v_{1}(x) \mathrm{d} x\right| \\
& \leq K_{\varepsilon}(t)\left|u_{1}\right|_{\Omega_{\varepsilon}^{\mathrm{a}}}\left|v_{1}\right|_{\Omega_{\varepsilon}^{\mathrm{a}}} \leq K_{\varepsilon}(t)\|u\|_{W}\|v\|_{W},
\end{aligned}
$$

since $\left|\partial_{t} J_{\varepsilon}^{\mathrm{a}}(x, t)\right| \leq K_{\varepsilon}(t)$ with $K_{\varepsilon} \in L^{1}(S)$ by lemma 4.1 .

Similarly, $J_{\varepsilon}^{\mathrm{a}}(x, \cdot) D_{\varepsilon}^{\mathrm{a}}(x, \cdot), D_{\varepsilon}^{\mathrm{w}}(x, \cdot)$ and $E_{\varepsilon}^{\mathrm{a}}(x, \cdot)$ are absolutely continuous and, therefore, $\mathcal{A}_{\varepsilon}(\cdot) u(v)$ is for arbitrary $u, v \in V$. Furthermore, we have

$$
\begin{aligned}
\left|\frac{\mathrm{d}}{\mathrm{d} t} \mathcal{A}_{\varepsilon}(t) u(v)\right| \leq & \int_{\Omega_{\varepsilon}^{\mathrm{a}}}\left|\partial_{t}\left(J_{\varepsilon}^{\mathrm{a}}(x, t) D_{\varepsilon}^{\mathrm{a}}(x, t)\right)\right|\left|\nabla u_{1}(x) \nabla v_{1}(x)\right| \mathrm{d} x \\
& +\varepsilon^{l} \int_{\Omega_{\varepsilon}^{\mathrm{w}}}\left|\partial_{t} D_{\varepsilon}^{\mathrm{w}}\right|\left|\nabla u_{2} \nabla v_{2}\right| \mathrm{d} x+\varepsilon^{l} \int_{\Omega_{\varepsilon}^{\mathrm{w}}}\left|\partial_{t} E_{\varepsilon}^{\mathrm{w}}\right|\left|\nabla u_{3} \nabla v_{3}\right| \mathrm{d} x \\
& +\varepsilon \int_{\Gamma_{\varepsilon}}\left|\partial_{t} a_{\varepsilon}\right|\left|\left(u_{1}-u_{2}\right)\left(v_{1}-v_{2}\right)\right| \mathrm{d} \sigma_{x}
\end{aligned}
$$

Since

$$
\left|\partial_{t}\left(J_{\varepsilon}^{\mathrm{a}}(x, t) D_{\varepsilon}^{\mathrm{a}}(x, t)\right)\right| \leq K_{\varepsilon}(t)\left\|D_{\varepsilon}^{\mathrm{a}}\right\|_{L^{\infty}\left(\Omega_{\varepsilon}^{\mathrm{a} \times S)}\right.}+\left\|J_{\varepsilon}^{\mathrm{a}}\right\|_{L^{\infty}\left(\Omega_{\varepsilon}^{\mathrm{a} \times S)}\right.}\left\|\partial_{t} D_{\varepsilon}^{\mathrm{a}}\right\|_{L^{\infty}(\Omega \times S)}
$$

and

$$
\begin{aligned}
& \varepsilon \int_{\Gamma_{\varepsilon}}\left|\partial_{t} a_{\varepsilon} \|\left(u_{1}-u_{2}\right)\left(v_{1}-v_{2}\right)\right| \mathrm{d} \sigma_{x} \\
& \leq \\
& \leq \varepsilon \partial_{t} a_{\varepsilon} \|_{L^{\infty}\left(\Gamma_{\varepsilon} \times S\right)}\left(\left|u_{1}\right|_{\Gamma_{\varepsilon}}+\left|u_{2}\right|_{\Gamma_{\varepsilon}}\right)\left(\left|v_{1}\right|_{\Gamma_{\varepsilon}}+\left|v_{2}\right|_{\Gamma_{\varepsilon}}\right) \\
& \leq C\left\|\partial_{t} a_{\varepsilon}\right\|_{L^{\infty}\left(\Gamma_{\varepsilon} \times S\right)}\left(\left(\left(\left|u_{1}\right|_{\Omega_{\varepsilon}^{\mathrm{a}}}+\left|u_{2}\right|_{\Omega_{\varepsilon}^{\mathrm{w}}}\right)+\varepsilon\left(\left|\nabla u_{1}\right|_{\Omega_{\varepsilon}^{\mathrm{a}}}+\left|\nabla u_{2}\right|_{\Omega_{\varepsilon}^{\mathrm{w}}}\right)\right)\right. \\
& \quad \times\left(\left(\left|v_{1}\right|_{\Omega_{\varepsilon}^{\mathrm{a}}}+\left|v_{2}\right|_{\Omega_{\varepsilon}^{\mathrm{w}}}\right)+\varepsilon\left(\left|\nabla v_{1}\right|_{\Omega_{\varepsilon}^{\mathrm{a}}}+\left|\nabla v_{2}\right|_{\Omega_{\varepsilon}^{\mathrm{w}}}\right)\right) \\
& \leq C_{\varepsilon}\|u\|_{V}\|v\|_{V},
\end{aligned}
$$

where we have made use of the trace lemma, (24) is also satisfied for $\mathcal{A}_{\varepsilon}(t)$ in $V$.

\section{Lemma 4.5}

The families of operators $\mathcal{A}_{\varepsilon}(t)$ and $\mathcal{B}_{\varepsilon}(t)$ defined by (28) and (30), respectively, satisfy (25) for $\mu=0$. For $\mu=1,(25)$ holds if one of the following two conditions is satisfied:

- $\partial_{t} J_{\varepsilon}^{\mathrm{a}}$ is non-negative. 
- $\partial_{t} J_{\varepsilon}^{\mathrm{a}} \in L^{\infty}(\Omega \times S)$.

Note that the latter condition is satisfied if $f_{\varepsilon}^{\mathrm{r}} \in L^{\infty}\left(S ; L^{2}(\Omega)\right)$.

Proof In order to show the estimate (25), we consider the elliptic term first. Let $v \in V$. Then, we have

$$
\begin{aligned}
2\left(\mathcal{A}_{\varepsilon}(t) v\right)(v)= & 2 \int_{\Omega_{\varepsilon}^{\mathrm{a}}} J_{\varepsilon}^{\mathrm{a}}(x, t) D_{\varepsilon}^{\mathrm{a}}(x, t)\left|\nabla v_{1}(x)\right|^{2} \mathrm{~d} x \\
& +2 \varepsilon^{l} \int_{\Omega_{\varepsilon}^{\mathrm{w}}} D_{\varepsilon}^{\mathrm{w}}(x, t)\left|\nabla v_{2}(x)\right|^{2} \mathrm{~d} x+\varepsilon^{l} \int_{\Omega_{\varepsilon}^{\mathrm{w}}} E_{\varepsilon}^{\mathrm{w}}(x, t)\left|\nabla v_{3}(x)\right|^{2} \mathrm{~d} x \\
& +2 \varepsilon \int_{\Gamma_{\varepsilon}} a_{\varepsilon}(x, t)\left|v_{1}(x)-v_{2}(x)\right|^{2} \mathrm{~d} \sigma_{x} \\
\geq & J_{\min }^{\mathrm{a}} D_{\min }^{\mathrm{a}}\left|\nabla v_{1}\right|_{\Omega_{\varepsilon}^{\mathrm{a}}}^{2}+\varepsilon^{l} D_{\min }^{\mathrm{w}}\left|\nabla v_{2}\right|_{\Omega_{\varepsilon}^{\mathrm{w}}}^{2}+\varepsilon^{l} E_{\min }^{\mathrm{w}}\left|\nabla v_{3}\right|_{\Omega_{\varepsilon}^{\mathrm{w}}}^{2} .
\end{aligned}
$$

Moreover, for $\lambda, \mu \geq 0$, we have

$$
\begin{aligned}
& \lambda\left(\mathcal{B}_{\varepsilon}(t) v\right)(v)=\lambda \int_{\Omega_{\varepsilon}^{\mathrm{a}}} J_{\varepsilon}^{\mathrm{a}}(x, t)\left|v_{1}(x)\right|^{2} \mathrm{~d} x+\lambda \int_{\Omega_{\varepsilon}^{\mathrm{w}}}\left|v_{2}(x)\right|^{2} \mathrm{~d} x+\lambda \int_{\Omega_{\varepsilon}^{\mathrm{w}}}\left|v_{3}(x)\right|^{2} \mathrm{~d} x, \\
& \mu\left(\mathcal{B}_{\varepsilon}^{\prime}(t) v\right)(v)=\mu \int_{\Omega_{\varepsilon}^{\mathrm{a}}} \partial_{t} J_{\varepsilon}^{\mathrm{a}}(x, t)\left|v_{1}(x)\right|^{2} \mathrm{~d} x .
\end{aligned}
$$

Therefore,

$$
\begin{aligned}
\lambda \mathcal{B}_{\varepsilon}(t) v(v) & +\mu \mathcal{B}_{\varepsilon}^{\prime}(t) v(v) \\
& \geq \int_{\Omega_{\varepsilon}^{\mathrm{a}}}\left(\lambda J_{\varepsilon}^{\mathrm{a}}(x, t)+\mu \partial_{t} J_{\varepsilon}^{\mathrm{a}}(x, t)\right)\left|v_{1}(x)\right|^{2} \mathrm{~d} x+\lambda\left|v_{2}\right|_{\Omega_{\varepsilon}^{\mathrm{w}}}^{2}+\lambda\left|v_{3}\right|_{\Omega_{\varepsilon}^{\mathrm{w}}}^{2} .
\end{aligned}
$$

Since $J_{\varepsilon}^{\mathrm{a}}(x, t)$ is positive and bounded away from zero, (25) is satisfied for $\mu=0$ and any positive $\lambda$.

Now, consider the case $\mu=1$. If we can then choose $\lambda$ large enough so that there exists a $\delta>0$ such that

$$
\lambda J_{\varepsilon}^{\mathrm{a}}(x, t)+\partial_{t} J_{\varepsilon}^{\mathrm{a}}(x, t) \geq \delta
$$

for a.e. $x$ and $t$, we can further estimate

$$
\lambda\left(\mathcal{B}_{\varepsilon}(t) v\right)(v)+\left(\mathcal{B}_{\varepsilon}^{\prime}(t) v\right)(v) \geq \delta\left|v_{1}\right|_{\Omega_{\varepsilon}^{\mathrm{a}}}^{2}+\lambda\left|v_{2}\right|_{\Omega_{\varepsilon}^{\mathrm{w}}}^{2}+\lambda\left|v_{3}\right|_{\Omega_{\varepsilon}^{\mathrm{w}}}^{2} .
$$

Then, (25) is satisfied. If $\partial_{t} J_{\varepsilon}^{\mathrm{a}} \geq 0$, it is clear that we can just choose $\lambda=1$. If this is not the case, we estimate as follows,

$$
\lambda J_{\varepsilon}^{\mathrm{a}}(x, t)+\partial_{t} J_{\varepsilon}^{\mathrm{a}}(x, t) \geq \lambda J_{\min }^{\mathrm{a}}-\left|\partial_{t} J_{\varepsilon}^{\mathrm{a}}(x, t)\right| \geq \lambda J_{\min }^{\mathrm{a}}-\left\|\partial_{t} J_{\varepsilon}^{\mathrm{a}}\right\|_{L^{\infty}(\Omega \times S)} .
$$

Choosing $\lambda>\left(\delta+\left\|\partial_{t} J_{\varepsilon}^{\mathrm{a}}\right\|_{L^{\infty}}\right) / J_{\min }^{\mathrm{a}}$ yields $(\dagger)$.

The above results can be summarized as follows. 


\section{Theorem 4.6}

For given $\varepsilon>0$, there exists a unique solution of problem (17).

Proof The existence of the solution to (17a) is given by lemma 4.1. The above considerations (particularly lemmas 4.4 and 4.5 ) show that the requirements of theorem 4.3 are satisfied which yields the existence and uniqueness of a solution for problem (17b)-(17d).

\subsection{Some $\varepsilon$-independent a-priori estimates}

We have already obtained the a-priori estimates (22) and (27). While the estimate (22) for $J_{\varepsilon}^{\mathrm{a}}$ is already $\varepsilon$-independent, this is not directly the case for the estimate $(27)$ on $u_{\varepsilon}^{\mathrm{a}}, u_{\varepsilon}^{\mathrm{w}}$ and $v_{\varepsilon}^{\mathrm{w}}$. This section is concerned with finding $\varepsilon$-independent estimates for the concentrations.

\section{Lemma 4.7}

The solution $\left(u_{\varepsilon}^{\mathrm{a}}, u_{\varepsilon}^{\mathrm{w}}, v_{\varepsilon}^{\mathrm{w}}\right) \in \mathcal{V}\left(\Omega_{\varepsilon}^{\mathrm{a}}\right) \times\left[\mathcal{V}\left(\Omega_{\varepsilon}^{\mathrm{w}}\right)\right]^{2}$ of problem $(17 \mathrm{~b})-(17 \mathrm{~d})$ satisfies

$$
\begin{gathered}
\left|u_{\varepsilon}^{\mathrm{a}}(t)\right|_{\Omega_{\varepsilon}^{\mathrm{a}}}+\left|\nabla u_{\varepsilon}^{\mathrm{a}}\right|_{\Omega_{\varepsilon}^{\mathrm{a}}, t}+\left|u_{\varepsilon}^{\mathrm{w}}(t)\right|_{\Omega_{\varepsilon}^{\mathrm{w}}}+\varepsilon^{l / 2}\left|\nabla u_{\varepsilon}^{\mathrm{w}}\right|_{\Omega_{\varepsilon}^{\mathrm{w}}, t}+\varepsilon^{1 / 2}\left|u_{\varepsilon}^{\mathrm{a}}-u_{\varepsilon}^{\mathrm{w}}\right|_{\Gamma_{\varepsilon}, t} \leq C \\
\left|v_{\varepsilon}^{\mathrm{w}}(t)\right|_{\Omega_{\varepsilon}^{\mathrm{w}}}+\varepsilon^{l / 2}\left|\nabla v_{\varepsilon}^{\mathrm{w}}\right|_{\Omega_{\varepsilon}^{\mathrm{w}}, t} \leq C
\end{gathered}
$$

for a.e. $t \in S$, where the constant $C$ depends on $T$ and the data but not on $\varepsilon$ if $\partial_{t} J_{\varepsilon}^{\mathrm{a}}$ is non-negative or bounded independently of $\varepsilon$ in $L^{\infty}(\Omega \times S)$.

Proof We begin with the equations for $u_{\varepsilon}^{\mathrm{a}}$ and $u_{\varepsilon}^{\mathrm{w}}$. Addition of equations $(17 \mathrm{~b})$ and $(17 \mathrm{c})$ gives

$$
\begin{array}{r}
\left(\partial_{t}\left(J_{\varepsilon}^{\mathrm{a}}(t) u_{\varepsilon}^{\mathrm{a}}(t)\right) \mid \phi^{\mathrm{a}}(t)\right)_{\Omega_{\varepsilon}^{\mathrm{a}}}+\left(\partial_{t} u_{\varepsilon}^{\mathrm{w}}(t) \mid \phi^{\mathrm{w}}(t)\right)_{\Omega_{\varepsilon}^{\mathrm{w}}}+\left(J_{\varepsilon}^{\mathrm{a}}(t) D_{\varepsilon}^{\mathrm{a}}(t) \nabla u_{\varepsilon}^{\mathrm{a}}(t) \mid \nabla \phi^{\mathrm{a}}(t)\right)_{\Omega_{\varepsilon}^{\mathrm{a}}} \\
+\varepsilon^{l}\left(D_{\varepsilon}^{\mathrm{w}}(t) \nabla u_{\varepsilon}^{\mathrm{w}}(t) \mid \nabla \phi^{\mathrm{w}}(t)\right)_{\Omega_{\varepsilon}^{\mathrm{w}}}+\varepsilon\left(a_{\varepsilon}(t)\left(u_{\varepsilon}^{\mathrm{a}}(t)-u_{\varepsilon}^{\mathrm{w}}(t)\right) \mid \phi^{\mathrm{a}}(t)-\phi^{\mathrm{w}}(t)\right)_{\Gamma_{\varepsilon}} \\
=-\left(r_{\varepsilon}^{\mathrm{u}}(t) f_{\varepsilon}^{\mathrm{r}}(t) \mid \phi^{\mathrm{w}}(t)\right)_{\Omega_{\varepsilon}^{\mathrm{w}}}
\end{array}
$$

Choosing the test function as

$$
\phi(x, t)= \begin{cases}u_{\varepsilon}^{\mathrm{a}}(x, t), & x \in \Omega_{\varepsilon}^{\mathrm{a}}, t \in(0, T), \\ u_{\varepsilon}^{\mathrm{w}}(x, t), & x \in \Omega_{\varepsilon}^{\mathrm{w}}, t \in(0, T),\end{cases}
$$

and noting that

$$
\left(\partial_{t}\left(J_{\varepsilon}^{\mathrm{a}}(t) u_{\varepsilon}^{\mathrm{a}}(t)\right) \mid u_{\varepsilon}^{\mathrm{a}}(t)\right)_{\Omega_{\varepsilon}^{\mathrm{a}}}=\frac{1}{2} \frac{\mathrm{d}}{\mathrm{d} t}\left(J_{\varepsilon}^{\mathrm{a}}(t) u_{\varepsilon}^{\mathrm{a}}(t) \mid u_{\varepsilon}^{\mathrm{a}}(t)\right)_{\Omega_{\varepsilon}^{\mathrm{a}}}+\frac{1}{2}\left(\partial_{t} J_{\varepsilon}^{\mathrm{a}}(t) u_{\varepsilon}^{\mathrm{a}}(t) \mid u_{\varepsilon}^{\mathrm{a}}(t)\right)_{\Omega_{\varepsilon}^{\mathrm{a}}}
$$


gives

$$
\begin{array}{r}
\frac{1}{2} \frac{\mathrm{d}}{\mathrm{d} t}\left(J_{\varepsilon}^{\mathrm{a}}(t) u_{\varepsilon}^{\mathrm{a}}(t) \mid u_{\varepsilon}^{\mathrm{a}}(t)\right)_{\Omega_{\varepsilon}^{\mathrm{a}}}+\left(\partial_{t} u_{\varepsilon}^{\mathrm{w}}(t) \mid u_{\varepsilon}^{\mathrm{w}}(t)\right)_{\Omega_{\varepsilon}^{\mathrm{w}}}+\left(J_{\varepsilon}^{\mathrm{a}}(t) D_{\varepsilon}^{\mathrm{a}}(t) \nabla u_{\varepsilon}^{\mathrm{a}}(t) \mid \nabla u_{\varepsilon}^{\mathrm{a}}(t)\right)_{\Omega_{\varepsilon}^{\mathrm{a}}} \\
+\varepsilon^{l}\left(D_{\varepsilon}^{\mathrm{w}}(t) \nabla u_{\varepsilon}^{\mathrm{w}}(t) \mid \nabla u_{\varepsilon}^{\mathrm{w}}(t)\right)_{\Omega_{\varepsilon}^{\mathrm{s}}}+\varepsilon\left(a_{\varepsilon}(t)\left(u_{\varepsilon}^{\mathrm{a}}(t)-u_{\varepsilon}^{\mathrm{w}}(t)\right) \mid u_{\varepsilon}^{\mathrm{a}}(t)-u_{\varepsilon}^{\mathrm{w}}(t)\right)_{\Gamma_{\varepsilon}} \\
=-\left(r_{\varepsilon}^{\mathrm{u}}(t) f_{\varepsilon}^{\mathrm{r}}(t) \mid u_{\varepsilon}^{\mathrm{w}}(t)\right)_{\Omega_{\varepsilon}^{\mathrm{w}}}-\frac{1}{2}\left(\partial_{t} J_{\varepsilon}^{\mathrm{a}}(t) u_{\varepsilon}^{\mathrm{a}}(t) \mid u_{\varepsilon}^{\mathrm{a}}(t)\right)_{\Omega_{\varepsilon}^{\mathrm{a}}}
\end{array}
$$

for a.e. $t$.

Integration with respect to time from 0 to $t$ gives

$$
\begin{aligned}
& \frac{1}{2}\left|\sqrt{J_{\varepsilon}^{\mathrm{a}}(t)} u_{\varepsilon}^{\mathrm{a}}(t)\right|_{\Omega_{\varepsilon}^{\mathrm{a}}}^{2}+\frac{1}{2}\left|u_{\varepsilon}^{\mathrm{w}}(t)\right|_{\Omega_{\varepsilon}^{\mathrm{w}}}^{2} \\
& +\left|\sqrt{J_{\varepsilon}^{\mathrm{a}} D_{\varepsilon}^{\mathrm{a}}} \nabla u_{\varepsilon}^{\mathrm{a}}\right|_{\Omega_{\varepsilon}^{\mathrm{a}}, t}^{2}+\varepsilon^{l}\left|\sqrt{D_{\varepsilon}^{\mathrm{w}}} \nabla u_{\varepsilon}^{\mathrm{w}}\right|_{\Omega_{\varepsilon}^{\mathrm{s}}}^{2}+\varepsilon\left|\sqrt{a_{\varepsilon}}\left(u_{\varepsilon}^{\mathrm{a}}-u_{\varepsilon}^{\mathrm{w}}\right)\right|_{\Gamma_{\varepsilon}, t}^{2} \\
& \quad=-\left(r_{\varepsilon}^{\mathrm{u}} f_{\varepsilon}^{\mathrm{r}} \mid u_{\varepsilon}^{\mathrm{w}}\right)_{\Omega_{\varepsilon}^{\mathrm{w}}, t}+\frac{1}{2}\left|\sqrt{J_{\varepsilon}^{\mathrm{a}}(0)} u_{\varepsilon}^{\mathrm{a}}(0)\right|_{\Omega_{\varepsilon}^{\mathrm{a}}}^{2}+\frac{1}{2}\left|u_{\varepsilon}^{\mathrm{w}}(0)\right|_{\Omega_{\varepsilon}^{\mathrm{w}}}^{2}-\frac{1}{2}\left(\partial_{t} J_{\varepsilon}^{\mathrm{a}} u_{\varepsilon}^{\mathrm{a}} \mid u_{\varepsilon}^{\mathrm{a}}\right)_{\Omega_{\varepsilon}^{\mathrm{a}}, t} .
\end{aligned}
$$

Making use of the assumptions on $J_{\varepsilon}^{\mathrm{a}}, D_{\varepsilon}^{\mathrm{a}}, D_{\varepsilon}^{\mathrm{w}}, a_{\varepsilon}$ and $r_{\varepsilon}^{\mathrm{u}}$ and utilizing the Cauchy-Schwarz and Young inequalities for the reaction term gives

$$
\begin{aligned}
& \frac{1}{2} J_{\min }^{\mathrm{a}}\left|u_{\varepsilon}^{\mathrm{a}}(t)\right|_{\Omega_{\varepsilon}^{\mathrm{a}}}^{2}+\frac{1}{2}\left|u_{\varepsilon}^{\mathrm{w}}(t)\right|_{\Omega_{\varepsilon}^{\mathrm{w}}}^{2}+J_{\min }^{\mathrm{a}} D_{\min }^{\mathrm{a}}\left|\nabla u_{\varepsilon}^{\mathrm{a}}\right|_{\Omega_{\varepsilon}^{\mathrm{a}}, t}^{2}+\varepsilon^{l} D_{\min }^{\mathrm{w}}\left|\nabla u_{\varepsilon}^{\mathrm{w}}\right|_{\Omega_{\varepsilon}^{\mathrm{s}}}^{2}+\varepsilon a_{\min }\left|u_{\varepsilon}^{\mathrm{a}}-u_{\varepsilon}^{\mathrm{w}}\right|_{\Gamma_{\varepsilon}, t}^{2} \\
\leq & \frac{1}{2}\left(r_{\max }^{\mathrm{u}}\right)^{2}\left|f_{\varepsilon}^{\mathrm{r}}\right|_{\Omega_{\varepsilon}^{\mathrm{w}}, t}^{2}+\frac{1}{2}\left|u_{\varepsilon}^{\mathrm{w}}\right|_{\Omega_{\varepsilon}^{\mathrm{w}}, t}^{2}+\frac{1}{2}\left|u_{\varepsilon}^{\mathrm{a}}(0)\right|_{\Omega_{\varepsilon}^{\mathrm{a}}}^{2}+\frac{1}{2}\left|u_{\varepsilon}^{\mathrm{w}}(0)\right|_{\Omega_{\varepsilon}^{\mathrm{w}}}^{2}+\frac{1}{2}|| \partial_{t} J_{\varepsilon}^{\mathrm{a}} \|_{L^{\infty}}\left|u_{\varepsilon}^{\mathrm{a}}\right|_{\Omega_{\varepsilon}^{\mathrm{a}}, t}^{2} .
\end{aligned}
$$

Note that if $\partial_{t} J_{\varepsilon}^{\mathrm{a}}$ is non-negative, the last term on the right-hand side can be omitted. Otherwise, the boundedness independently of $\varepsilon$ is required. Collection of all constants,

$$
\begin{aligned}
& \left|u_{\varepsilon}^{\mathrm{a}}(t)\right|_{\Omega_{\varepsilon}^{\mathrm{a}}}^{2}+\left|u_{\varepsilon}^{\mathrm{w}}(t)\right|_{\Omega_{\varepsilon}^{\mathrm{w}}}^{2}+\left|\nabla u_{\varepsilon}^{\mathrm{a}}\right|_{\Omega_{\varepsilon}^{\mathrm{a}}, t}^{2}+\varepsilon^{l}\left|\nabla u_{\varepsilon}^{\mathrm{w}}\right|_{\Omega_{\varepsilon}^{\mathrm{w}}, t}^{2}+\varepsilon\left|u_{\varepsilon}^{\mathrm{a}}-u_{\varepsilon}^{\mathrm{w}}\right|_{\Gamma_{\varepsilon}, t}^{2} \\
& \quad \leq C\left(\left|f_{\varepsilon}^{\mathrm{r}}\right|_{\Omega_{\varepsilon}^{\mathrm{w}}, t}^{2}+\left|u_{\varepsilon}^{\mathrm{a}}\right|_{\Omega_{\varepsilon}^{\mathrm{w}}, t}^{2}+\left|u_{\varepsilon}^{\mathrm{w}}\right|_{\Omega_{\varepsilon}^{\mathrm{w}}, t}^{2}+\left|u_{\varepsilon}^{\mathrm{a}}(0)\right|_{\Omega_{\varepsilon}^{\mathrm{a}}}^{2}+\left|u_{\varepsilon}^{\mathrm{w}}(0)\right|_{\Omega_{\varepsilon}^{\mathrm{w}}}^{2}\right),
\end{aligned}
$$

and application of Gronwall's inequality gives (32a).

Testing of equation $(17 \mathrm{~d})$ with $v_{\varepsilon}^{\mathrm{w}}$ and integration with respect to time gives

$$
\frac{1}{2}\left|v_{\varepsilon}^{\mathrm{w}}(t)\right|_{\Omega_{\varepsilon}^{\mathrm{w}}}^{2}+\varepsilon^{l}\left|\nabla v_{\varepsilon}^{\mathrm{w}}\right|_{\Omega_{\varepsilon}^{\mathrm{w}}, t}^{2}=\left(r_{\varepsilon}^{\mathrm{v}} f_{\varepsilon}^{\mathrm{r}} \mid v_{\varepsilon}^{\mathrm{w}}\right)_{\Omega_{\varepsilon}^{\mathrm{w}}, t}+\frac{1}{2}\left|v_{0}^{\mathrm{w}}\right|_{\Omega_{\varepsilon}^{\mathrm{w}}}^{2} .
$$

Utilizing the Cauchy-Schwarz and Young inequalities for the reaction term and application of Gronwall's inequality gives (32b).

\subsection{Convergence}

In this section, we want to investigate the convergence of the sequences of solutions as $\varepsilon \rightarrow 0$. We use the notion of two-scale convergence. For details concerning classical results we refer to [18], [19], [20], [21], [22] and [23]. For simplicity, we discuss the two-scale convergence for sequences independent of time. Since time is only a parameter with respect to two-scale convergence, this is no restriction, cf. [24], e.g. 


\section{Definition 4.8 (Two-scale convergence)}

A sequence of functions $v_{\varepsilon}$ in $L^{2}(\Omega)$ is said to two-scale converge to a limit function $v_{0}(x, y) \in L^{2}(\Omega \times Y)$ iff

$$
\lim _{\varepsilon \rightarrow 0} \int_{\Omega} v_{\varepsilon}(x) \phi(x, x / \varepsilon) \mathrm{d} x=\int_{\Omega} \int_{Y} v_{0}(x, y) \phi(x, y) \mathrm{d} y \mathrm{~d} x
$$

for all $\phi \in C_{0}^{\infty}\left(\Omega ; C_{\#}^{\infty}(Y)\right)$ where the subscript \# denotes periodicity. A sequence of functions $v_{\varepsilon}$ in $L^{2}\left(\Gamma_{\varepsilon}\right)$ is said to two-scale converge to a limit function $v_{0}(x, y) \in L^{2}(\Omega \times \Gamma)$ iff

$$
\lim _{\varepsilon \rightarrow 0} \varepsilon \int_{\Gamma_{\varepsilon}} v_{\varepsilon}(x) \phi(x, x / \varepsilon) \mathrm{d} \sigma_{x}=\int_{\Omega} \int_{\Gamma} v_{0}(x, y) \phi(x, y) \mathrm{d} \sigma_{y} \mathrm{~d} x
$$

for all $\phi \in C_{0}^{\infty}\left(\Omega ; C_{\#}^{\infty}(Y)\right)$.

The following theorem is fundamental to the notion of two-scale convergence (cf. theorem 1 in [18] or theorem 1.2 in [19]). In particular, it applies to $J_{\varepsilon}^{\mathrm{a}}$.

\section{Theorem 4.9}

Let $u_{\varepsilon}$ be a bounded sequence in $L^{2}(\Omega)$. Then, there exists a subsequence such that $u_{\varepsilon}$ two-scale converges to a limit function $u_{0} \in L^{2}(\Omega \times Y)$.

The a-priori estimates (32) ensure that standard two-scale convergence results apply to the sequences $u_{\varepsilon}^{\mathrm{a}}, u_{\varepsilon}^{\mathrm{w}}$ and $v_{\varepsilon}^{\mathrm{w}}$, whose limits we denote by $u^{\mathrm{a}}, u^{\mathrm{w}}$ and $v^{\mathrm{w}}$, respectively. Therefore, we only state the results required here. The proofs can be found in [19, 25], e.g. For the formulation of the next theorem, the following notation is introduces: For a function $v^{\alpha} \in L^{2}\left(\Omega_{\varepsilon}^{\alpha}\right)$, its zero extension to $\Omega$ is denoted by $\tilde{v}^{\alpha}$. Clearly: $\tilde{v}^{\alpha} \in L^{2}(\Omega)$.

\section{Theorem 4.10}

Let $\alpha \in\{\mathrm{a}, \mathrm{w}\}, 0 \leq \lambda \leq 2$ and $u^{\alpha} \in W^{1,2}\left(\Omega_{\varepsilon}^{\alpha}\right)$ satisfy the estimate

$$
\left|u_{\varepsilon}^{\alpha}\right|_{\Omega_{\varepsilon}^{\alpha}}+\varepsilon^{\lambda / 2}\left|\nabla u_{\varepsilon}^{\alpha}\right|_{\Omega_{\varepsilon}^{\alpha}} \leq C,
$$

with $C$ independent of $\varepsilon$. Then, the following statements hold:

(a) Let $\lambda=0$ and $\Omega_{\varepsilon}^{\alpha}$ be connected. Then, there exist limit functions $u^{\alpha} \in$ $W^{1,2}(\Omega)$ as well as $u_{1}^{\alpha} \in L^{2}\left(\Omega ; W_{\#}^{1,2}(Y) / \mathbb{R}\right)$ such that for a subsequence the following convergence results hold in two-scale sense:

$$
\tilde{u}_{\varepsilon}^{\alpha} \longrightarrow \chi^{\alpha} u^{\alpha} \text { and } \widetilde{\nabla_{x}} u_{\varepsilon}^{\alpha} \longrightarrow \chi^{\alpha}\left(\nabla_{x} u^{\alpha}+\nabla_{y} u_{1}^{\alpha}\right)
$$

(b) Let $0<\lambda<2$. Then, there exists limit functions $u^{\alpha} \in L^{2}(\Omega)$ and $u_{1}^{\alpha} \in$ $L^{2}\left(\Omega ; W_{\#}^{1,2}(Y) / \mathbb{R}\right)$ such that, at least for a subsequence, the following convergence results hold in two-scale sense:

$$
\tilde{u}_{\varepsilon}^{\alpha} \longrightarrow \chi^{\alpha} u^{\alpha} \quad \text { and } \quad \varepsilon^{\lambda / 2} \widetilde{\nabla_{x}} u_{\varepsilon}^{\alpha} \longrightarrow \chi^{\alpha} \nabla_{y} u_{1}^{\alpha}
$$


(c) Let $\lambda=2$. Then, there exists a limit function $u^{\alpha} \in L^{2}\left(\Omega ; W_{\#}^{1,2}(Y)\right)$ such that, at least for a subsequence, the following convergence results hold in two-scale sense:

$$
\tilde{u}_{\varepsilon}^{\alpha} \longrightarrow \chi^{\alpha} u^{\alpha} \quad \text { and } \quad \varepsilon \widetilde{\nabla_{x}} u_{\varepsilon}^{\alpha} \longrightarrow \chi^{\alpha} \nabla_{y} u^{\alpha}
$$

(d) In any of the above cases the trace of $u_{\varepsilon}^{\alpha}$ on $\Gamma_{\varepsilon}$ two-scale converges to the trace of the limit function on $\Gamma$ in the sense of (34).

We also cite a result stating when the product of two two-scale convergent sequences converges to the product of their limits (cf. theorem 1.8 of [19]):

\section{Theorem 4.11}

Assume that $u_{\varepsilon}$ and $v_{\varepsilon}$ are two bounded sequences of functions in $L^{2}(\Omega)$ which two-scale converge to limits $u_{0}$ and $v_{0}$ in $L^{2}(\Omega \times Y)$, respectively. Assume further that

$$
\lim _{\varepsilon \rightarrow 0}\left|u_{\varepsilon}\right|_{\Omega}=\left|u_{0}\right|_{\Omega \times Y}
$$

Then, we have

$$
u_{\varepsilon} v_{\varepsilon} \longrightarrow \int_{Y} u_{0}(x, y) v_{0}(x, y) \mathrm{d} y
$$

weakly in $C_{0}^{\infty}(\Omega)^{\prime}$.

A sufficient condition for (39) to hold, is that $u_{\varepsilon}$ is a sum of functions belonging to the following classes,

- functions being continuous with respect to one space variable,

- functions being a product of functions which depend on one space variable and time only.

An analogous result holds for sequences given on $\Gamma_{\varepsilon}$, cf. [20], where condition (39) needs to be replaced by

$$
\lim _{\varepsilon \rightarrow 0} \varepsilon\left|u_{\varepsilon}\right|_{\Gamma_{\varepsilon}}^{2}=\left|u_{0}\right|_{\Omega \times \Gamma}^{2} .
$$

\section{$5 \quad$ Identification of the limit problems}

Let $f^{\mathrm{r}}(x, y, t)$ be the two-scale limit of $f_{\varepsilon}^{\mathrm{r}}(x, t)$ in $L^{2}(\Omega \times S)$. Theorem 4.9 ensures that, at least for a subsequence, $J_{\varepsilon}^{\mathrm{a}}$ two-scale converges to a limit function $J^{\text {a }}$ while theorem 4.10 yields the existence of two-scale limit functions $u^{\mathrm{a}}, u^{\mathrm{w}}$ and $v^{\mathrm{w}}$ of $u_{\varepsilon}^{\mathrm{a}}, u_{\varepsilon}^{\mathrm{w}}$ and $v_{\varepsilon}^{\mathrm{w}}$, respectively.

We begin with the limit problem for $J_{\varepsilon}^{\mathrm{a}}$. 


\section{Proposition 5.1}

The limit function $J^{\mathrm{a}}$ of $J_{\varepsilon}^{\mathrm{a}}$ as $\varepsilon \rightarrow 0$ satisfies (20a).

Proof Choosing the test function in (17a) as $\varphi^{\mathrm{J}}(x, y, t)$ with $\varphi^{\mathrm{J}} \in L^{2}(\Omega \times$ $Y \times S)$ and integrating over $Y$, we obtain

$$
\begin{aligned}
\int_{0}^{T} \int_{\Omega} \int_{Y} \mathcal{T}_{\varepsilon}\left(\partial_{t} J_{\varepsilon}^{\mathrm{a}}\right)(x, t) \varphi^{\mathrm{J}}(x, y, t) \mathrm{d} y \mathrm{~d} x \mathrm{~d} t \\
=-C^{\mathrm{m}} \int_{0}^{T} \int_{\Omega} \int_{Y} \int_{Z^{\mathrm{w}}} \mathcal{T}_{\varepsilon}\left(f_{\varepsilon}^{\mathrm{r}}\right)(x, z, t) \mathrm{d} z \varphi^{\mathrm{J}}(x, y, t) \mathrm{d} y \mathrm{~d} x \mathrm{~d} t,
\end{aligned}
$$

where we have used the fact that $J_{\varepsilon}^{\mathrm{a}}$ is constant in each cell, and, therefore, $\partial_{t} J_{\varepsilon}^{\mathrm{a}}=\mathcal{T}_{\varepsilon}\left(\partial_{t} J_{\varepsilon}^{\mathrm{a}}\right)$ is independent of $y$. Since the sequences are independent of $y$, the limit equation as $\varepsilon \rightarrow 0$ is given by

$$
\begin{aligned}
\int_{0}^{T} \int_{\Omega} \partial_{t} J^{\mathrm{a}}(x, t) & \int_{Y} \varphi^{\mathrm{J}}(x, y, t) \mathrm{d} y \mathrm{~d} x \mathrm{~d} t \\
& =-C^{\mathrm{m}} \int_{0}^{T} \int_{\Omega} \int_{Z^{\mathrm{w}}} f^{\mathrm{r}}(x, z, t) \mathrm{d} z \int_{Y} \varphi^{\mathrm{J}}(x, y, t) \mathrm{d} y \mathrm{~d} x \mathrm{~d} t .
\end{aligned}
$$

By periodic unfolding, these are also the two-scale limits and, moreover, it suffices to take $\varphi^{\mathrm{J}}$ independent of $y$. Therefore,

$$
\int_{0}^{T} \int_{\Omega} \partial_{t} J^{\mathrm{a}}(x, t) \varphi^{\mathrm{J}}(x, t) \mathrm{d} x \mathrm{~d} t=-C^{\mathrm{m}} \int_{0}^{T} \int_{\Omega} \int_{Z^{\mathrm{w}}} f^{\mathrm{r}}(x, y, t) \mathrm{d} y \varphi^{\mathrm{J}}(x, t) \mathrm{d} x \mathrm{~d} t
$$

for all $\varphi^{\mathrm{J}} \in L^{2}(\Omega \times S)$.

\section{Proposition 5.2}

The limit function $u^{\mathrm{a}}$ associated with the sequence of solutions $u_{\varepsilon}^{\mathrm{a}}$ satisfies the weak macromodel equation (20b).

PROOF Integration of the weak micromodel equation (17b) for $u_{\varepsilon}^{\mathrm{a}}$ with respect to the time variable, integration by parts in the time-derivative term and choosing the test function to be of the form

$$
\phi(x, y, t)=\phi_{0}(x, t)+\varepsilon \phi_{1}(x, x / \varepsilon, t)
$$

with $\left(\phi_{0}, \phi_{1}\right) \in C_{0}^{\infty}\left(S ; C^{\infty}(\Omega)\right) \times C_{0}^{\infty}\left(S ; C^{\infty}\left(\Omega ; C_{\#}^{\infty}(Y)\right)\right)$ gives

$$
\begin{aligned}
& -\int_{S \times \Omega} J_{\varepsilon}^{\mathrm{a}}(x, t) u_{\varepsilon}^{\mathrm{a}}(x, t) \chi^{\mathrm{a}}(x / \varepsilon) \partial_{t}\left[\phi_{0}(x, t)+\varepsilon \phi_{1}(x, x / \varepsilon, t)\right] \mathrm{d} x \mathrm{~d} t \\
& +\int_{S \times \Omega} J_{\varepsilon}^{\mathrm{a}}(x, t) D^{\mathrm{a}}(x, x / \varepsilon, t) \nabla_{x} u_{\varepsilon}^{\mathrm{a}}(x, t) \chi^{\mathrm{a}}(x / \varepsilon) \\
& \quad \times\left[\nabla_{x} \phi_{0}(x, t)+\varepsilon \nabla_{x} \phi_{1}(x, x / \varepsilon, t)+\nabla_{y} \phi_{1}(x, x / \varepsilon, t)\right] \mathrm{d} x \mathrm{~d} t \\
& +\varepsilon \int_{S \times \Gamma_{\varepsilon}} a(x, x / \varepsilon, t)\left[u_{\varepsilon}^{\mathrm{a}}(x, t)-u_{\varepsilon}^{\mathrm{w}}(x, t)\right]\left[\phi_{0}(y, t)+\varepsilon \phi_{1}(x, x / \varepsilon, t)\right] \mathrm{d} \sigma_{x} \mathrm{~d} t=0 .
\end{aligned}
$$


The limit as $\varepsilon$ tends to zero is now determined. The functions $u^{\mathrm{a}}$ and $u_{1}^{\mathrm{a}}$ appearing in this proof are always understood as the limit functions provided by proposition 5.1 and theorem 4.10 .

The limits of the three terms in $(\dagger)$ can be computed individually. For the first two terms, we note that the fact that the limit of the product is equal to the product of the limits follows from theorem 4.11 as well as the fact that $J_{\varepsilon}^{a}$ does not depend on the microvariable. The third term can be handled using part (d) theorem 4.10 .

Putting everything together,

$$
\begin{aligned}
& \left|Z^{\mathrm{a}}\right| \int_{S \times \Omega} \partial_{t}\left(J^{\mathrm{a}}(x, t) u^{\mathrm{a}}(x, t)\right) \phi_{0}(x, t) \mathrm{d} x \mathrm{~d} t \\
& +\int_{S \times \Omega} \int_{Z^{\mathrm{a}}} J^{\mathrm{a}}(x, t) D^{\mathrm{a}}(x, y, t)\left(\nabla_{x} u^{\mathrm{a}}(x, t)+\nabla_{y} u_{1}^{\mathrm{a}}(x, y, t)\right) \\
& \quad \times\left[\nabla_{x} \phi_{0}(x, t)+\nabla_{y} \phi_{1}(x, y, t)\right] \mathrm{d} y \mathrm{~d} x \mathrm{~d} t \\
& +\int_{S \times \Omega} \int_{\Gamma} a(x, y, t)\left(u^{\mathrm{a}}(x, t)-u^{\mathrm{w}}(x, y, t)\right) \mathrm{d} \sigma_{y} \phi_{0}(x, t) \mathrm{d} x \mathrm{~d} t=0
\end{aligned}
$$

for all $\left(\phi_{0}, \phi_{1}\right)$ is obtained, where we have already integrated by parts again in the time-derivative term. Also, note that $u^{\mathrm{w}}$ does not depend on $y$ if $\lambda<2$. Choosing $\phi_{0} \equiv 0$ yields

$\int_{S \times \Omega} \int_{Z^{\mathrm{a}}} J^{\mathrm{a}}(x, t) D^{\mathrm{a}}(x, y, t)\left(\nabla_{x} u^{\mathrm{a}}(x, t)+\nabla_{y} u_{1}^{\mathrm{a}}(x, y, t)\right) \nabla_{y} \phi_{1}(x, y, t) \mathrm{d} y \mathrm{~d} x \mathrm{~d} t=0$ for all $\phi_{1} \in C_{0}^{\infty}\left(S ; C^{\infty}\left(\Omega ; C_{\#}^{\infty}(Y)\right)\right)$. Assuming $u_{1}^{\mathrm{a}}=\sum_{j=1}^{N} \partial_{x_{j}} u^{\mathrm{a}}(x, t) \varsigma_{j}^{\mathrm{a}}(x, y, t)$, the equation is satisfied if $\varsigma_{j}^{\mathrm{a}}$ is the solution of the cell problem (18) because

$$
\begin{aligned}
0= & \int_{S \times \Omega} \int_{Z^{\mathrm{a}}} J^{\mathrm{a}}(x, t) D^{\mathrm{a}}(x, y, t) \\
& \times\left[\sum_{j=1}^{N} \partial_{x_{j}} u^{\mathrm{a}}(x, t) \nabla_{y} \varsigma_{j}^{\mathrm{a}}(x, y, t) \nabla_{y} \phi_{1}(x, y, t)+\nabla_{x} u^{\mathrm{a}}(x, t) \nabla_{y} \phi_{1}(x, y, t)\right] \mathrm{d} y \mathrm{~d} x \mathrm{~d} t \\
= & \int_{S \times \Omega} \int_{Z^{\mathrm{a}}} J^{\mathrm{a}}(x, t) D^{\mathrm{a}}(x, y, t) \\
& \times\left[-\sum_{j=1}^{N} \partial_{x_{j}} u^{\mathrm{a}}(x, t) e_{j} \nabla_{y} \phi_{1}(x, y, t)+\nabla_{x} u^{\mathrm{a}}(x, t) \nabla_{y} \phi_{1}(x, y, t)\right] \mathrm{d} y \mathrm{~d} x \mathrm{~d} t,
\end{aligned}
$$

which is obviously true for all $\phi_{1}$. On the other hand, if $\varsigma_{j}^{\mathrm{a}}$ is the solution of the cell problem (18), the equation is satisfied if $u_{1}^{\mathrm{a}}=\sum_{j=1}^{N} \partial_{x_{j}} u^{\mathrm{a}}(x, t) \varsigma_{j}^{\mathrm{a}}(x, y, t)$.

Choosing $\phi_{1} \equiv 0$ gives

$$
\begin{aligned}
& \left|Z^{\mathrm{a}}\right| \int_{S \times \Omega} \partial_{t}\left(J^{\mathrm{a}}(x, t) u^{\mathrm{a}}(x, t)\right) \phi_{0}(x, t) \mathrm{d} x \mathrm{~d} t \\
& +\int_{S \times \Omega} \int_{Z^{\mathrm{a}}} J^{\mathrm{a}}(x, t) D^{\mathrm{a}}(x, y, t)\left(\nabla_{x} u^{\mathrm{a}}(x, t)+\nabla_{y} u_{1}^{\mathrm{a}}(x, y, t)\right) \nabla_{x} \phi_{0}(x, t) \mathrm{d} y \mathrm{~d} x \mathrm{~d} t
\end{aligned}
$$




$$
+\int_{S \times \Omega} \int_{\Gamma} a(x, y, t)\left(u^{\mathrm{a}}(x, t)-u^{\mathrm{w}}(x, y, t)\right) \mathrm{d} \sigma_{y} \phi_{0}(x, t) \mathrm{d} x \mathrm{~d} t=0
$$

for all $\phi_{0} \in C_{0}^{\infty}\left(S ; C^{\infty}(\Omega)\right)$. Using $u_{1}^{\mathrm{a}}=\sum_{j=1}^{N} \partial_{x_{j}} u^{\mathrm{a}}(x, t) \varsigma_{j}^{\mathrm{a}}(x, y, t)$, the second term can be rewritten,

$$
\begin{array}{r}
\int_{S \times \Omega} \int_{Z^{\mathrm{a}}} J^{\mathrm{a}}(x, t) D^{\mathrm{a}}(x, y, t)\left(\nabla_{x} u^{\mathrm{a}}(x, t)+\nabla_{y} u_{1}^{\mathrm{a}}(x, y, t)\right) \nabla_{x} \phi_{0}(x, t) \mathrm{d} y \mathrm{~d} x \mathrm{~d} t \\
=\int_{S \times \Omega} P^{\mathrm{a}}(x, t) \nabla_{x} u^{\mathrm{a}}(x, t) \nabla_{x} \phi_{0}(x, t) \mathrm{d} x \mathrm{~d} t
\end{array}
$$

where the tensor $P^{\mathrm{a}}$ is defined in (20c). Thus,

$$
\begin{aligned}
& \left|Z^{\mathrm{a}}\right| \int_{S \times \Omega} \partial_{t}\left(J^{\mathrm{a}}(x, t) u^{\mathrm{a}}(x, t)\right) \phi_{0}(x, t) \mathrm{d} x \mathrm{~d} t \\
& +\int_{S \times \Omega} P^{\mathrm{a}}(x, t) \nabla_{x} u^{\mathrm{a}}(x, t) \nabla_{x} \phi_{0}(x, t) \mathrm{d} x \mathrm{~d} t \\
& \quad=-\int_{S \times \Omega} \int_{\Gamma} a(x, y, t)\left(u^{\mathrm{a}}(x, t)-u^{\mathrm{w}}(x, y, t)\right) \mathrm{d} \sigma_{y} \phi_{0}(x, t) \mathrm{d} x \mathrm{~d} t
\end{aligned}
$$

for all $\phi_{0} \in C_{0}^{\infty}\left(S ; C^{\infty}(\Omega)\right)$.

\section{Proposition 5.3}

The limit functions $u^{\mathrm{w}}$ and $v^{\mathrm{w}}$ associated with the sequences of solutions $u_{\varepsilon}^{\mathrm{w}}$ and $v_{\varepsilon}^{\mathrm{w}}$, respectively, satisfy the weak macromodel equations (20d) and (20e).

Proof The proof can be obtained analogously to that of proposition 5.2. For $\lambda<2$, the test function needs to be chosen as

$$
\phi(x, t)=\phi_{0}(x, t)+\varepsilon^{1-\lambda / 2} \phi_{1}(x, x / \varepsilon, t)
$$

with $\left(\phi_{0}, \phi_{1}\right) \in C_{0}^{\infty}\left(S ; C^{\infty}(\Omega)\right) \times C_{0}^{\infty}\left(S ; C^{\infty}\left(\Omega ; C_{\#}^{\infty}(Y)\right)\right)$ while, for $\lambda \geq 2$, $\phi(x, t)=\phi_{1}(x, x / \varepsilon, t)$ is the appropriate test function.

\section{Acknowledgements}

The author would like to thank the German National Academic Foundation for the financial support and the Special Priority Program 1122 "Prediction of the course of physico-chemical damage processes involving mineral materials" of the German Research Foundation for the insights in concrete carbonation. Moreover, fruitful discussions with M. Böhm and S. A. Meier from the University of Bremen and W. Jäger from the University of Heidelberg are greatly appreciated. 


\section{References}

[1] J. Kropp, Relations between transport characteristics and durability, in: J. Kropp, H. K. Hilsdorf (Eds.), Performance criteria for concrete durability, RILEM Report 12, E \& FN SPON, 1995, pp. 97-137.

[2] T. A. Bier, Karbonatisierung und Realkalisierung von Zementstein und Beton, PhD dissertation, University of Karlsruhe (1988).

[3] S. A. Meier, M. A. Peter, M. Böhm, A two-scale modelling approach to reaction-diffusion processes in porous materials, Comp. Mat. Sci. 39 (1) (2007) 29-34.

[4] S. A. Meier, M. A. Peter, A. Muntean, M. Böhm, J. Kropp, A twoscale approach to concrete carbonation, in: R. M. Ferreirad, J. Gulikers, C. Andrade (Eds.), RILEM Proc. PRO 56: Proc. Int. RILEM Workshop on Integral Service Life Modelling of Concrete Structures, 2007, pp. 3-10.

[5] M. A. Peter, Homogenisation in domains with evolving microstructure, C. R. Mécanique 335 (7) (2007) 357-362.

[6] C. Conca, J. I. Díaz, C. Timofte, Effective chemical processes in porous media, Math. Mod. Meth. Appl. Sci. 13 (10) (2003) 1437-1462.

[7] M. A. Peter, M. Böhm, Scalings in homogenisation of reaction, diffusion and interfacial exchange in a two-phase medium, in: M. Fila, A. Handlovicova, K. Mikula, M. Medved, P. Quittner, D. Sevcovic (Eds.), Proc. Equadiff-11, 2005, pp. 369-376.

[8] M. A. Peter, Coupled reation-diffusion processes and evolving microstructure: mathematical modelling and homongenisation, PhD dissertation, University of Bremen, also: Logos Verlag Berlin, 2007. (2006).

[9] D. Cioranescu, A. Damlamian, G. Griso, Periodic unfolding and homogenization, C. R. Acad. Sci. Paris, Ser. I 335 (2002) 99-104.

[10] J. D. Logan, Transport modeling in hydrogeochemical systems, Springer, 2001.

[11] A. Muntean, A moving-boundary problem: modeling, analysis and simulation of concrete carbonation, PhD dissertation, University of Bremen, also: Cuvillier, 2006. (2006).

[12] K. Wilmański, Thermomechanics of continua, Springer, 1998.

[13] J. Bear, Dynamics of fluids in porous media, Elsevier, 1972.

[14] J. Bear, Y. Bachmat, Introduction to modeling of transport phenomena in porous media, Kluwer, 1990.

[15] M. A. Peter, Homogenisation of a chemical degradation mechanism inducing an evolving microstructure, C. R. Mécanique 335 (11) (2007) 679-684.

[16] R. E. Showalter, Monotone operators in Banach space and nonlinear partial differential equations, American Mathematical Society, 1997.

[17] G. W. Clark, R. E. Showalter, Two-scale convergence of a model for flow in a partially fissured medium, Electronic J. Differential Equations 1999 (2) (1999) 1-20.

[18] G. Nguetseng, A general convergence result for a functional related to the theory of homogenization, SIAM J. Math. Anal. 20 (3) (1989) 608-629. 
[19] G. Allaire, Homogenization and two-scale convergence, SIAM J. Math. Anal. 23 (6) (1992) 1482-1518.

[20] G. Allaire, A. Damlamian, U. Hornung, Two-scale convergence on periodic surfaces and applications, in: A. P. Bourgeat, C. Carasso, S. Luckhaus, A. Mikelić (Eds.), Proceedings of the international conference on mathematical modelling of flow through porous media, World Scientific, 1995, pp. 15-25.

[21] M. Neuss-Radu, Some extensions of two-scale convergence, C. R. Acad. Sci. Paris, Ser. I 322 (1996) 899-904.

[22] G. Allaire, M. Briane, Multiscale convergence and reiterated homogenisation, Proc. Roy. Soc. Edinb. 126A (1996) 297-342.

[23] D. Lukkassen, G. Nguetseng, P. Wall, Two-scale convergence, Int. J. Pure Appl. Math. 2 (1) (2002) 35-86.

[24] A. Holmbom, Homogenization of parabolic equations an alternative approach and some corrector-type results, Appl. Math. 42 (5) (1997) 321343.

[25] M. A. Peter, M. Böhm, Different choices of scaling in homogenization of diffusion and interfacial exchange in a porous medium, Math. Meth. Appl. Sci.. (in press). 